\title{
Light stops, blind spots, and isospin violation in the MSSM
}

\author{
Andreas Crivellin, ${ }^{a, b}$ Martin Hoferichter, ${ }^{c, d, b}$ Massimiliano Procura ${ }^{e, b}$ and \\ Lewis C. Tunstall ${ }^{b}$ \\ ${ }^{a}$ CERN Theory Division, \\ CH-1211 Geneva 23, Switzerland \\ ${ }^{b}$ Albert Einstein Center for Fundamental Physics, \\ Institute for Theoretical Physics, University of Bern, \\ Sidlerstrasse 5, CH-3012 Bern, Switzerland \\ ${ }^{c}$ Institut für Kernphysik, Technische Universität Darmstadt, \\ Schlossgartenstraße 2, D-64289 Darmstadt, Germany \\ ${ }^{d}$ ExtreMe Matter Institute EMMI, GSI Helmholtzzentrum für Schwerionenforschung GmbH, \\ Planckstraße 1, D-64291 Darmstadt, Germany \\ ${ }^{e}$ Fakultät für Physik, Universität Wien, \\ Boltzmanngasse 5, A-1090 Vienna, Austria \\ E-mail: andreas.crivellin@cern.ch, \\ hoferichter@theorie.ikp.physik.tu-darmstadt.de, \\ mprocura@univie.ac.at, tunstall@itp.unibe.ch
}

ABSTRACT: In the framework of the MSSM, we examine several simplified models where only a few superpartners are light. This allows us to study WIMP-nucleus scattering in terms of a handful of MSSM parameters and thereby scrutinize their impact on dark matter direct-detection experiments. Focusing on spin-independent WIMP-nucleon scattering, we derive simplified, analytic expressions for the Wilson coefficients associated with Higgs and squark exchange. We utilize these results to study the complementarity of constraints due to direct-detection, flavor, and collider experiments. We also identify parameter configurations that produce (almost) vanishing cross sections. In the proximity of these so-called blind spots, we find that the amount of isospin violation may be much larger than typically expected in the MSSM. This feature is a generic property of parameter regions where cross sections are suppressed, and highlights the importance of a careful analysis of the nucleon matrix elements and the associated hadronic uncertainties. This becomes especially relevant once the increased sensitivity of future direct-detection experiments corners the MSSM into these regions of parameter space.

Keywords: Supersymmetry Phenomenology

ArXiv EPrint: 1503.03478 


\section{Contents}

1 Introduction 1

2 Theoretical preliminaries 3

2.1 Spin-independent neutralino-nucleus cross section: scalar matrix elements 3

2.2 Simplified expressions for spin-independent scattering of bino-like dark matter 7

3 Simplified models: blind spots and isospin violation $\quad 11$

$\begin{array}{lll}3.1 & \text { SM-like Higgs exchange } & 14\end{array}$

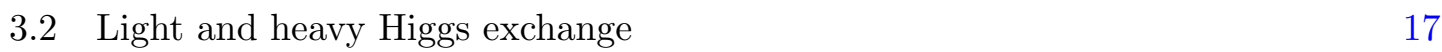

3.3 SM-like Higgs and light squark exchange 20

3.4 Generic Higgs and light squark exchange 23

4 Conclusions $\quad 28$

$\begin{array}{ll}\text { A Perturbative diagonalization of the neutralino mass matrix } & 29\end{array}$

B Analytic expressions for $\chi$-nucleon scattering $\quad 30$

\section{Introduction}

Establishing the microscopic nature of Dark Matter (DM) is one of the central, open questions in cosmology and particle physics. In the context of cold nonbaryonic DM, the prevailing paradigm is based on weakly interacting massive particles (WIMPs), and extensive theoretical and experimental resources have been devoted towards identifying viable candidates and developing methods to detect them. One of the most studied WIMPs arises in the Minimal Supersymmetric Standard Model (MSSM), where an assumed $R$ parity ensures that the lightest superpartner (LSP) is a stable neutralino $\chi$ composed of bino $\tilde{B}$, wino $\tilde{W}$, and Higgsino $\tilde{H}$ eigenstates. The mass of the LSP is expected to lie in the range of tens to hundreds of $\mathrm{GeV}$.

In its general form, however, the MSSM contains more than 100 parameters, most of which are tied to the hidden sector which breaks supersymmetry (SUSY) at some scale $M_{\mathrm{SUSY}}$. Since these parameters are unknown a priori, it is necessary to restrict the dimensionality of the parameter space in order to obtain a predictive framework with which to undertake phenomenological analyses. One way to achieve this is to adopt a specific mechanism that describes high-scale SUSY-breaking in terms of a small number of parameters. For example, the constrained MSSM (CMSSM) with minimal supergravity [1-4] only involves five free parameters, but faces increasing tension [5-12] with the non-observation of superpartners at the LHC experiments and other observables like the measured Higgs 
mass and anomalous magnetic moment of the muon. Alternatively, one can remain agnostic about the features of SUSY-breaking and incorporate data-driven constraints, as in e.g. the $\mathrm{p}$ (henomenological)MSSM [13, 14], where only 19 free parameters are used to capture the essential features of weak-scale SUSY.

In both approaches, long computational chains involving spectrum generators, the calculation of decay rates, or the DM relic abundance are typically required in order to explore the relevant parameter space. This strategy has been used extensively for the CMSSM [512 ] and pMSSM [15-18] to analyze $\chi$-nucleon scattering and impose limits from current DM direct-detection experiments such as SuperCDMS [19], XENON100 [20], and LUX [21], as well as upcoming proposals like XENON1T [22], LUX-ZEPLIN (LZ) [23], and SuperCDMS SNOLAB [24]. While these parameter scans allow one to gain useful information about the status of the theory in light of global fits, they generally hinder attempts to clearly identify which contributions associated with the underlying theory parameters can have the greatest impact on a signal of interest. An analytical understanding of the underlying parameter space can instead be obtained in the context of so-called simplified models, defined $^{1}[30]$ to be minimal theories of weak-scale SUSY where all but a handful of the superpartners relevant for DM phenomenology are decoupled from the spectrum.

For spin-independent (SI) $\chi$-nucleon scattering, the choice of simplified model is guided by the dominant contributions to the cross section, namely, Higgs and squark exchange [3136]. To date, the focus has largely concerned the role of the Higgs sector, both in the decoupling limit where a single SM-like Higgs $h$ is present in the spectrum [30,37], or in the more general case $[38,39]$ where the heavier $C P$-even Higgs $H$ is included. This focus is chiefly motivated by the fact that current bounds on the masses of gluinos and (degenerate) squarks of the first two generations are larger than about $1 \mathrm{TeV}$ [40, 41], and so their contribution to the SI cross section can be safely ignored. ${ }^{2}$

However, the decoupling of third-generation squarks - especially stops — upsets the main motivation behind the MSSM, namely, its ability to stabilize the electroweak scale $v \simeq 174 \mathrm{GeV}$ against loop corrections in a technically natural fashion [46-51]. In other words, if naturalness is to remain a useful criterion with which to constrain the MSSM parameter space, then the spectrum should (minimally) include light stops $\tilde{t}_{1,2}$ and - due to $\mathrm{SU}(2)_{L}$ invariance - a left-handed sbottom $\tilde{b}_{L}[46,52-54]$. While the search for top and bottom squarks remains a primary focus of the LHC experiments, their impact on DM direct-detection limits has not been explored in detail.

The purpose of this paper is to present an analytical scheme which allows one to successively include those states which are most relevant for naturalness and DM direct detection. In particular, we consider a bino-like LSP and derive simplified, analytic expressions for the Wilson coefficients associated with SI scattering. We examine in detail the contributions from Higgs and third-generation squark exchange, and study the interplay of collider, flavor, and DM constraints. As in previous analyses of the Higgs sector [30, 3739], our scheme allows us to identify so-called blind spots in parameter space, where the

\footnotetext{
${ }^{1}$ For a definition of "simplified models" in the context of LHC searches, see [25-29].

${ }^{2}$ However, for non-degenerate squarks [42, 43] the constraints from FCNCs are satisfied [44], and the collider bounds are significantly weakened [45]. In this case, contributions from the first two generations could also be important for SI $\chi$-nucleon scattering.
} 
SI cross section is strongly suppressed by either a particular set of parameters [30, 37], or destructive interference $[38,39]$ in the scattering amplitude. This effect was first identified numerically through a scan of the CMSSM parameter space [55-57], while lower bounds on the $\chi$-nucleon cross sections were first discussed in [58] for both the CMSSM and a generalized MSSM framework. A key feature of our analysis is that in the vicinity of blind spots, the amount of isospin violation may be much larger than typically expected for the MSSM [59]. ${ }^{3}$ In order to account for isospin-violating effects originating from the nucleon matrix elements of the scalar quark currents, we use the formalism developed in [66], which provides an accurate determination of the hadronic uncertainties.

The paper is organized as follows. In section 2 we establish our notation for $\chi$-nucleus scattering and comment on the differing treatments $[59,66,67]$ of the nucleon scalar matrix elements found in the literature. The leading MSSM contributions to the Wilson coefficients are then identified using a systematic expansion in $v / M_{\mathrm{SUSY}}$ which generates simplified analytic expressions for the Wilson coefficients associated with Higgs and squark exchange. Section 3 examines four simplified models, where, driven by naturalness, we successively include the most relevant particles as active degrees of freedom. In each case, we discuss the conditions for blind spots and examine the amount of isospin violation allowed by current and projected limits from SI DM scattering. Our analysis shows that the absence of DM signals pushes the MSSM into regions of parameter space where isospin-violating effects are likely to become relevant.

\section{Theoretical preliminaries}

\subsection{Spin-independent neutralino-nucleus cross section: scalar matrix elements}

We start by providing some definitions for the elastic scattering of the lightest neutralino $\chi$ off a species of nucleus $\mathcal{N}={ }_{Z}^{A} X$, where $Z$ and $A$ denote the atomic and mass numbers respectively. Typically, the dependence of the cross section on the small momentum transfer is assumed to be described by nuclear form factors. At zero momentum transfer and for one-body currents only, the cross section for $\chi \mathcal{N} \rightarrow \chi \mathcal{N}$ is given by

$$
\sigma_{\mathrm{SI}}=\frac{4 \mu_{\chi}^{2}}{\pi}\left[Z f_{p}+(A-Z) f_{n}\right]^{2}
$$

Here, $\mu_{\chi}=m_{\chi} m_{\mathcal{N}} /\left(m_{\chi}+m_{\mathcal{N}}\right)$ is the reduced mass of the $\chi \mathcal{N}$ system, while $f_{p}$ and $f_{n}$ are effective (zero-momentum) SI couplings of the LSP to the proton and neutron respectively.

For nucleons $N$, the $\chi-N$ couplings $f_{N}$ are defined by

$$
\frac{f_{N}}{m_{N}}=\sum_{q=u, d, s} f_{q}^{N} C_{q}+f_{Q}^{N} \sum_{q=c, b, t} C_{q}, \quad N=p \text { or } n,
$$

where $C_{q}$ is the Wilson coefficient of the scalar operator $\bar{m}_{q} \bar{\chi} \chi \bar{q} q$ with running quark mass $\bar{m}_{q}$, and

$$
m_{N} f_{q}^{N}=\left\langle N\left|\bar{m}_{q} \bar{q} q\right| N\right\rangle, \quad f_{Q}^{N}=\frac{2}{27}\left(1-f_{u}^{N}-f_{d}^{N}-f_{s}^{N}\right) .
$$

\footnotetext{
${ }^{3}$ Large isospin violation has been put forward as a mechanism to reconcile contradictory DM directdetection signal claims and null observations [60-65].
} 
The coefficients $f_{q}^{N}$ can be interpreted as the fraction of the nucleon mass generated by the respective quark scalar current and are often referred to as nucleon scalar couplings. In the framework adopted in (2.3), the heavy quarks $c, b, t$ are integrated out, so that, via the trace anomaly [68-71] of the QCD energy-momentum tensor, their scalar coefficients $f_{Q}^{N}$ can be expressed in terms of the light-quark ones [35]. As shown by Drees and Nojiri [72], this procedure fails if the squarks are sufficiently light, and in section 2 we discuss the necessary modifications to (2.2) which account for the exact one-loop result.

We note that (2.3) holds at leading order in $\alpha_{s}$ : in the case of the charm quark, this may not be sufficiently accurate, so that either higher-order corrections [73-75] or a nonperturbative determination on the lattice could become mandatory. Similarly, corrections to the single-nucleon picture underlying (2.1) in the form of two-nucleon currents can be systematically taken into account using effective field theory $[64,65,76,77]$. In this paper, we use (2.1) and (2.3) to investigate the amount of isospin violation that can be generated within several simplified models, given the hadronic uncertainties of the single-nucleon coefficients $f_{q}^{N}$ for the light quarks $u, d, s$.

Traditionally, the scalar matrix elements of the light quarks have been determined from a combination of chiral $\mathrm{SU}(3)_{L} \times \mathrm{SU}(3)_{R}$ perturbation theory $\left(\chi \mathrm{PT}_{3}\right)$ and phenomenological input inferred from the pion-nucleon $\sigma$-term $\sigma_{\pi N}$ and the hadron mass spectrum $[55,59,64,78]$. A central feature of this approach is that the up- and down-quark coefficients $f_{u, d}^{N}$ are reconstructed from two three-flavor quantities: the so-called strangeness content of the nucleon

$$
y=\frac{2\langle N|\bar{s} s| N\rangle}{\langle N|\bar{u} u+\bar{d} d| N\rangle}=\frac{2 f_{s}^{N} / \bar{m}_{s}}{f_{u}^{N} / \bar{m}_{u}+f_{d}^{N} / \bar{m}_{d}},
$$

and another parameter

$$
z=\frac{\langle N|\bar{u} u-\bar{s} s| N\rangle}{\langle N|\bar{d} d-\bar{s} s| N\rangle}=\frac{f_{u}^{N} / \bar{m}_{u}-f_{s}^{N} / \bar{m}_{s}}{f_{d}^{N} / \bar{m}_{d}-f_{s}^{N} / \bar{m}_{s}}
$$

that is related to isospin violation. As a result, the inherent uncertainties of $\chi \mathrm{PT}_{3}$ (typically of order $30 \%$ ) propagate to the two-flavor sector. Furthermore, $z$ is usually extracted from a leading-order fit to baryon masses [79], and this compounds the problem of obtaining reliable uncertainty estimates.

For the up- and down-quark coefficients $f_{u, d}^{N}$, these problems can be circumvented by using the two-flavor theory $\chi \mathrm{PT}_{2}$ directly, thus avoiding the three-flavor expansion in the first place [66]. Starting from the $\chi \mathrm{PT}_{2}$ expansion of the nucleon mass at third chiral order and including the effects due to strong isospin violation, one finds

$$
\begin{aligned}
f_{u}^{N} & =\frac{\sigma_{\pi N}(1-\xi)}{2 m_{N}}+\Delta f_{u}^{N}, & f_{d}^{N} & =\frac{\sigma_{\pi N}(1+\xi)}{2 m_{N}}+\Delta f_{d}^{N}, \\
\Delta f_{u}^{p} & =(1.0 \pm 0.2) \times 10^{-3}, & \Delta f_{u}^{n} & =(-1.0 \pm 0.2) \times 10^{-3}, \\
\Delta f_{d}^{p} & =(-2.1 \pm 0.4) \times 10^{-3}, & \Delta f_{d}^{n} & =(2.0 \pm 0.4) \times 10^{-3},
\end{aligned}
$$

where the $\sigma$-term is defined as $\sigma_{\pi N} \equiv\langle N|\hat{m}(\bar{u} u+\bar{d} d)| N\rangle$, averaged over proton and neutron, $\hat{m}=\left(\bar{m}_{u}+\bar{m}_{d}\right) / 2$, and

$$
\xi=\frac{\bar{m}_{d}-\bar{m}_{u}}{\bar{m}_{d}+\bar{m}_{u}}=0.36 \pm 0.04
$$


is taken from [80].

For the present work, one particularly important aspect of the $\chi \mathrm{PT}_{2}$ approach [66] is that isospin violation can be rigorously accounted for, including uncertainty estimates. This aspect can be nicely illustrated by considering the differences

$$
f_{u}^{p}-f_{u}^{n}=(1.9 \pm 0.4) \times 10^{-3} \quad \text { and } \quad f_{d}^{p}-f_{d}^{n}=(-4.1 \pm 0.7) \times 10^{-3},
$$

wherein the terms $\sigma_{\pi N}(1 \pm \xi) / 2 m_{N}$ from (2.6) cancel. ${ }^{4}$ Using the $\chi \mathrm{PT}_{3}$ approach, these differences are overestimated by roughly a factor of 2 , as in e.g. [67]:

$$
f_{u}^{p}-f_{u}^{n}=4.3 \times 10^{-3}, \quad f_{d}^{p}-f_{d}^{n}=-8.2 \times 10^{-3} .
$$

Alternatively, one could introduce further measures of isospin violation like $f_{u}^{p}-f_{d}^{n}$ and $f_{u}^{n}-f_{d}^{p}$ (motivated by the quark-model picture of the nucleon), but these combinations depend on the specific value of $\sigma_{\pi N}$. In the isospin-conserving limit, all up- and downcoefficients obtained from the chiral expansion of the nucleon mass become equal $f_{u}^{p}=$ $f_{u}^{n}=f_{d}^{p}=f_{d}^{n}=\sigma_{\pi N} / 2 m_{N}$, so that the relations $f_{u}^{p}=f_{d}^{n}$ and $f_{u}^{n}=f_{d}^{p}$ are fulfilled.

Ultimately, the quantities relevant for the direct-detection cross section are the parameters defined in (2.2), after multiplication by the Wilson coefficients and summing over quark flavors. In particular, the cross section (2.1) may be rewritten as

$$
\sigma_{\mathrm{SI}}=\frac{4 \mu_{\chi}^{2}}{\pi} f_{p}^{2}\left[A+(A-Z)\left(\frac{f_{n}}{f_{p}}-1\right)\right]^{2}
$$

so that the departure of $f_{n} / f_{p}$ from unity emerges as a convenient measure of isospin violation. In this context, care has to be taken in interpreting the limits on the WIMPnucleon cross section $\sigma_{\text {SI }}^{p, n}$ given by experimental collaborations. Indeed, these are generally extracted via the relation

$$
\sigma_{\mathrm{SI}}=\sigma_{\mathrm{SI}}^{N}\left(\frac{\mu_{\chi}}{\mu_{N}}\right)^{2} A^{2}
$$

where $\mu_{N}$ is the reduced mass of the $\chi$-nucleon system. We stress that $\sigma_{\mathrm{SI}}^{p}$ in (2.11) can be identified with the SI $\chi$-proton cross section only under the assumption $f_{n} \simeq f_{p}$. If isospinviolating effects are large, it is natural to compare against the $\chi$-nucleus cross section (2.1) directly, and (2.11) indicates how the experimental limits are to be rescaled.

In general, the effects of isospin violation depend on the target nucleus. For the mass range $m_{\chi} \simeq 50-1000 \mathrm{GeV}$ considered in most of our analysis (section 3), the strongest limits on SI $\chi$-nucleon scattering are currently set by LUX [21]. In the context of isospin violation, this prompts us to focus on the projected reach of upcoming xenon-based experiments like XENON1T [22] and LZ [23]. However, this raises the question whether other experiments like SuperCDMS SNOLAB [24] (based on germanium) can be used to place complementary constraints on $f_{n} / f_{p}$. To quantify the difference between xenon-based constraints and other

\footnotetext{
${ }^{4}$ The chiral expansion of the nucleon mass difference $m_{p}-m_{n}$ is known to have a large chiral logarithm at fourth order, with coefficient $\left(6 g_{\mathrm{A}}^{2}+1\right) / 2 \approx 5[81,82]$. We have checked that including this logarithm in the analysis leads to changes of the $\Delta f_{u, d}^{N}$ well within the uncertainties given in (2.6).
} 


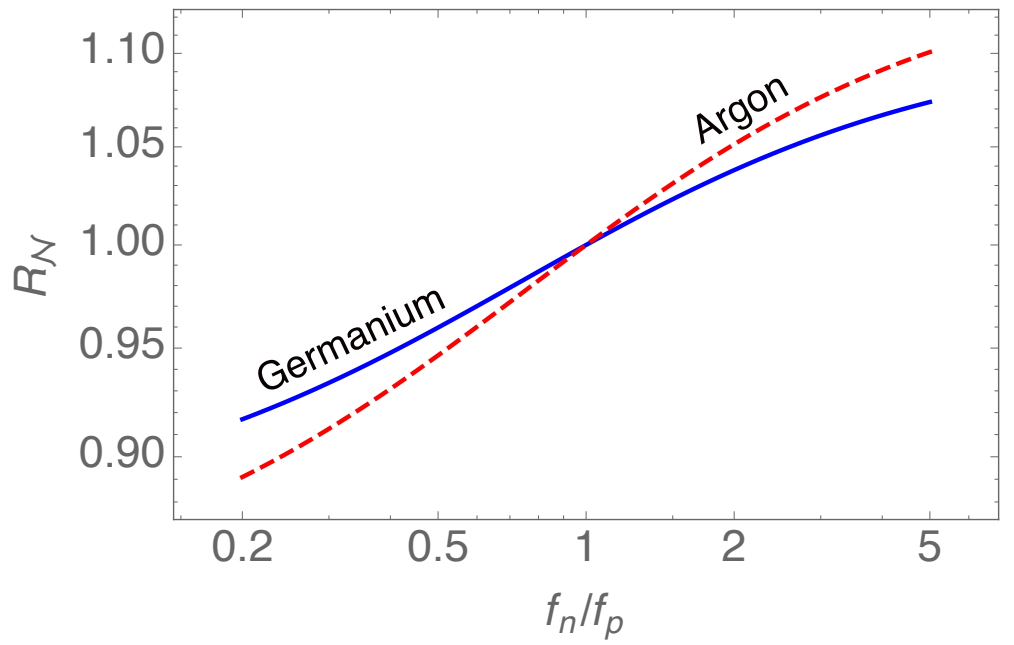

Figure 1. Relative difference $R_{\mathcal{N}}$ (defined in (2.12)) between xenon and other nuclear targets $\mathcal{N}$ as a function of the isospin violation measure $f_{n} / f_{p}$. The dashed line in red shows the comparison against $\mathcal{N}=$ germanium, while the solid blue line corresponds to the $\mathcal{N}=$ argon comparison.

nuclei, consider the $f_{n} / f_{p}$ dependence of the ratio

$$
R_{\mathcal{N}}=\frac{\sigma_{\mathrm{SI}}^{\mathrm{Xe}}}{\sigma_{\mathrm{SI}}^{\mathcal{N}}}\left(\frac{\mu_{\chi}^{\mathcal{N}} A_{\mathcal{N}}}{\mu_{\chi}^{\mathrm{Xe}} A_{\mathrm{Xe}}}\right)^{2},
$$

normalized such that $R_{\mathcal{N}}=1$ in the isospin-conserving limit. For SI scattering off argon and germanium, the result is shown in figure 1, where we observe a maximum difference of around $10 \%$ for $f_{n} / f_{p}$ much larger or smaller than unity. In the simplified models considered in section 3 , the difference between $f_{n}$ and $f_{p}$ is generated entirely by SM quantities, so the improved limits offered by e.g. SuperCDMS SNOLAB are limited to the percent level. Moreover, the location of blind spots is determined by the condition $f_{n, p} \simeq 0$, so neither the blind spot nor the uncertainty on $f_{n} / f_{p}$ depends significantly on the atomic/mass numbers of the nuclear target.

The crucial input quantity $\sigma_{\pi N}$ is not yet precisely determined: in section 3 we show the dependence on this parameter explicitly in the case of generic Higgs exchange, and later fix its central value to $\sigma_{\pi N}=50 \mathrm{MeV}$ for illustrative purposes. The need for a precise determination of $\sigma_{\pi N}$ has triggered many ongoing efforts, including lattice-QCD calculations at (nearly) physical values of the pion mass; see [67, 83-85] for a compilation of recent results and improved phenomenological analyses. The challenge in the phenomenological approach, i.e. extracting $\sigma_{\pi N}$ from $\pi N$ scattering, lies in controlling the required analytic continuation of the isoscalar $\pi N$ amplitude into the unphysical region [86], which might even be sensitive to isospin-breaking corrections $[87,88]$. This analytic continuation can be stabilized with the help of the low-energy data that have become available in recent years thanks to accurate pionic-atom measurements [89, 90], leading to a precise extraction of the $\pi N$ scattering lengths [91, 92]. A systematic analysis of $\pi N$ scattering based on this input as well as constraints from unitarity, analyticity, and crossing symmetry along the lines 
of [93-95] will help clarify the situation concerning the phenomenological determination of $\sigma_{\pi N}[96-98]$.

In our numerical analysis, we compare three different methods used to determine the scalar couplings $f_{q}^{N}$ and their uncertainties:

- Method 1: based on $\chi \mathrm{PT}_{2}[66]$, with $f_{u, d}^{N}$ determined from (2.6) and $f_{s}^{N}$ from lattice QCD. It is well known [99] that the $\chi$-nucleon cross section is sensitive to the value of $f_{s}^{N}$. In our analysis we adopt the lattice average from [85]:

$$
f_{s}^{N}=0.043 \pm 0.011
$$

- Method 2: corresponds to the traditional $\chi \mathrm{PT}_{3}$ approach $[55,59,64,78]$, where $f_{u, d}^{N}$ and $f_{s}^{N}$ are determined via the three-flavor quantities $y$ and $z$. In this approach, the strange-quark scalar matrix element is defined via

$$
f_{s}^{N}=\frac{\sigma_{\pi N}}{2 m_{N}} \frac{\bar{m}_{s}}{\hat{m}} y
$$

where $\bar{m}_{s} / \hat{m}=(27.4 \pm 0.4)$ [80], the strangeness content is taken from the relation $y=$ $1-\sigma_{0} / \sigma_{\pi N}$, with $\sigma_{0}=(36 \pm 7) \mathrm{MeV}[100]$, and $z \simeq 1.49$ is extracted from leading-order fits to the baryon mass spectrum [79]. This approach introduces uncertainties that are difficult to quantify and is sensitive to the precise value of $\sigma_{\pi N}$. The range $\sigma_{\pi N}=$ $(50 \pm 15) \mathrm{MeV}$ covering the determinations discussed above [83, 84, 96, 97] translates to $f_{s}^{N}=0.2 \pm 0.2$. Even for moderate values of the $\sigma$-term, large values $f_{s}^{N} \approx 0.25$ have been inferred in this way. Such large values are incompatible with recent lattice calculations, which provide a more reliable determination of $f_{s}^{N}$ (see (2.13) for a recent average). A determination of the uncertainty bands arising from this approach requires us to attach an error to $z$, which, as argued before, is impossible to quantify reliably. Therefore, based on general expectations for the convergence pattern in $\chi \mathrm{PT}_{3}$, we simply attach to $z$ a $30 \%$ error. ${ }^{5}$

- Method 3: corresponds to the implementation in MICROMEGAs-4.1.2 [67] and follows the traditional approach in Method 2, but with (2.14) inverted so that $y$ is a function of $f_{s}^{N}$. With lattice QCD input (2.13) for $f_{s}^{N}$, this method has reduced uncertainties compared with Method 2, but suffers from the fact that $f_{u, d}^{N}$ still depend on the three-flavor quantities $y$ and $z$.

\subsection{Simplified expressions for spin-independent scattering of bino-like dark matter}

Let us now derive analytic expressions for SI $\chi$-nucleon scattering in the MSSM. We first review the complete expressions due to tree-level Higgs and squark exchange, and then simplify them by expanding in powers of $v / M_{\text {SUSY }}$. For light third-generation squarks, a procedure [102] to extend our results to include the one-loop corrections [72] is discussed below.

\footnotetext{
${ }^{5}$ This is consistent with an analysis [101] of the quark-mass dependence of octet baryons.
} 
The lightest neutralino is a linear combination of $\tilde{B}, \tilde{W}$, and $\tilde{H}_{u, d}$ interaction eigenstates,

$$
\chi \equiv \tilde{\chi}_{1}^{0}=Z_{11}^{\chi} \tilde{B}+Z_{21}^{\chi} \tilde{W}+Z_{31}^{\chi} \tilde{H}_{d}+Z_{41}^{\chi} \tilde{H}_{u},
$$

while the neutralino mass matrix is given by

$$
M^{\chi}=\left(\begin{array}{cccc}
M_{1} & 0 & -\frac{1}{2} g_{1} v_{d} & \frac{1}{2} g_{1} v_{u} \\
0 & M_{2} & \frac{1}{2} g_{2} v_{d} & -\frac{1}{2} g_{2} v_{u} \\
-\frac{1}{2} g_{1} v_{d} & \frac{1}{2} g_{2} v_{d} & 0 & -\mu \\
\frac{1}{2} g_{1} v_{u} & -\frac{1}{2} g_{2} v_{u} & -\mu & 0
\end{array}\right) .
$$

Here $M_{1}\left(M_{2}\right)$ are the soft SUSY-breaking masses of the bino (wino), $\mu$ is the Higgsino mass parameter, and $v_{u, d}$ are the two Higgs $H_{u, d}$ vacuum expectation values, whose ratio $v_{u} / v_{d}$ is denoted by $\tan \beta$. Note that while $v_{u, d}$ can be rendered real and positive by an appropriate phase redefinition of the Higgs fields, $M_{1}$ and $M_{2}$ are in general complex if the gluino mass is assumed to be real (as is standard convention). The neutralino mixing in (2.15) is determined by the unitary matrix $Z^{\chi}$ which diagonalizes $M^{\chi}$ [103]:

$$
Z_{I^{\prime} I}^{\chi} M_{I^{\prime} J^{\prime}}^{\chi} Z_{J^{\prime} J}^{\chi}=\delta_{I J} m_{\tilde{\chi}_{I}^{0}}
$$

In the squark sector, the squared masses $m_{\tilde{q}_{s}}^{2}$ are eigenvalues of the $6 \times 6$ matrices in flavor/chirality space,

$$
\begin{aligned}
\mathcal{M}_{\tilde{u}}^{2} & =\left(\begin{array}{cc}
V^{\dagger} \mathbf{m}_{Q}^{2} V+v_{u}^{2} \mathbf{Y}_{u} \mathbf{Y}_{u}^{\dagger}+g_{u_{L}} m_{Z}^{2} c_{2 \beta} & -v_{u}\left(\mathbf{Y}_{u} \mathbf{A}_{u}+\mathbf{Y}_{u} \mu \cot \beta\right) \\
-v_{u}\left(\mathbf{A}_{u}^{\dagger} \mathbf{Y}_{u}^{\dagger}+\mathbf{Y}_{u}^{\dagger} \mu^{*} \cot \beta\right) & \mathbf{m}_{U}^{2}+v_{u}^{2} \mathbf{Y}_{u}^{\dagger} \mathbf{Y}_{u}+g_{u_{R}} m_{Z}^{2} c_{2 \beta}
\end{array}\right), \\
\mathcal{M}_{\tilde{d}}^{2} & =\left(\begin{array}{cc}
\mathbf{m}_{Q}^{2}+v_{d}^{2} \mathbf{Y}_{d} \mathbf{Y}_{d}^{\dagger}+g_{d_{L}} m_{Z}^{2} c_{2 \beta} & -v_{d}\left(\mathbf{Y}_{d} \mathbf{A}_{d}+\mathbf{Y}_{d} \mu \tan \beta\right) \\
-v_{d}\left(\mathbf{A}_{d}^{\dagger} \mathbf{Y}_{d}^{\dagger}+\mathbf{Y}_{d}^{\dagger} \mu^{*} \tan \beta\right) & \mathbf{m}_{D}^{2}+v_{d}^{2} \mathbf{Y}_{d}^{\dagger} \mathbf{Y}_{d}+g_{d_{R}} m_{Z}^{2} c_{2 \beta}
\end{array}\right) .
\end{aligned}
$$

Here, the soft SUSY-breaking squark masses are $\mathbf{m}_{Q}, \mathbf{m}_{U}$, and $\mathbf{m}_{D}, \mathbf{Y}_{u, d}$ are complex Yukawa matrices, $V$ is the CKM matrix, and we have assumed flavor universality $\mathbf{a}_{q}=$ $\mathbf{Y}_{q} \mathbf{A}_{q}$ for the trilinear $A$-terms. We also use $s$ and $c$ for sine and cosine, so that $s_{\beta} \equiv \sin \beta$, $c_{2 \beta} \equiv \cos 2 \beta$, etc. The weak neutral-current couplings

$$
g_{q}=I_{3}^{q}-e_{q} s_{W}^{2}
$$

are defined in terms of the third component of weak isospin $I_{3}^{q}$, electric charge $e_{q}$, and $s_{W}^{2} \simeq 0.2231$. A unitary transformation

$$
Z_{s^{\prime} s}^{\tilde{q} *}\left(\mathcal{M}_{\tilde{q}}^{2}\right)_{s^{\prime} t^{\prime}} Z_{t^{\prime} t}^{\tilde{q}}=m_{\tilde{q}_{s}}^{2} \delta_{s t}
$$

gives the physical basis with diagonal squark mass matrices, where we adopt the convention to order the states in increasing mass. We have also defined the super-CKM basis in (2.18) as the one with diagonal (and in general, complex) Yukawa couplings $Y_{q_{i}}$ [104].

We have now introduced the necessary ingredients to discuss $\chi$-quark scattering in the MSSM. The tree-level contributions to the Wilson coefficients $C_{q_{i}}$ are the diagrams shown in figure 2. For $C P$-conserving neutralino interactions, these amplitudes were calculated long ago in [31-35], and extended in [105] to include $C P$-violating effects. 

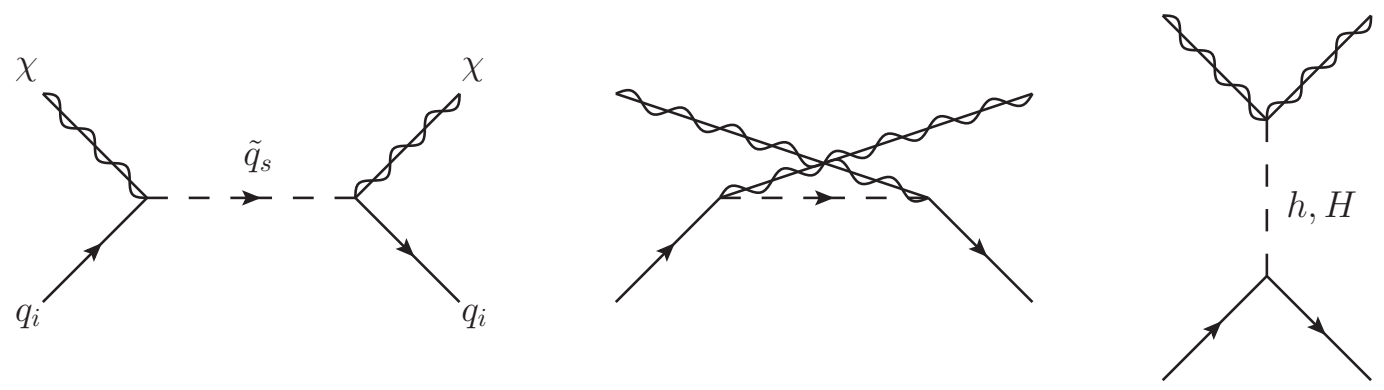

Figure 2. Tree-level MSSM graphs which contribute to the SI cross section for $\chi$-quark scattering.

In our conventions, the contributions to $C_{q_{i}}$ due to squark exchange in the $s$ - and $u$-channels at zero momentum transfer $\operatorname{read}^{6}$

$$
\bar{m}_{q_{i}} C_{q_{i}}^{\tilde{q}}=\frac{1}{8} \sum_{s=1}^{6}\left[\frac{1}{\left(m_{\chi}+m_{q_{i}}\right)^{2}-m_{\tilde{q}_{s}}^{2}+i \epsilon}+\frac{1}{\left(m_{\chi}-m_{q_{i}}\right)^{2}-m_{\tilde{q}_{s}}^{2}+i \epsilon}\right] \operatorname{Re}\left\{\Gamma_{L}^{q_{i} \tilde{q}_{s} *} \Gamma_{R}^{q_{i} \tilde{q}_{s}}\right\},
$$

where there is no sum over $i$, and a pole mass $m_{q_{i}}$ enters in the squark propagator. Since we work at $M_{\mathrm{SUSY}}$, the running quark masses $\bar{m}_{q_{i}}$ must also be evaluated at this scale. For the Higgs-exchange contribution we have ${ }^{7}$

$$
\bar{m}_{q_{i}} C_{q_{i}}^{h, H}=\frac{1}{2} \sum_{k=1}^{2} \frac{1}{m_{H_{k}}^{2}} \operatorname{Re}\left\{\Gamma_{\chi \chi}^{H_{k}}\right\} \operatorname{Re}\left\{\Gamma_{q_{i} q_{i}}^{H_{k}}\right\}
$$

where $H_{1} \equiv H$ and $H_{2} \equiv h$. We assume that $m_{h} \simeq 125 \mathrm{GeV}$ is the mass of the Higgslike resonance found at the LHC $[106,107]$ and $m_{h}<m_{H}$. In general, the $\chi q_{i} \tilde{q}_{s}$ and $H_{k}^{0} \chi \chi$ couplings appearing in (2.21) and (2.22) are complicated expressions involving the mixing matrices $Z^{\chi}$ and $Z^{\tilde{q}}$. Thus the SI cross section is typically determined numerically. However, it is known [108-110] that one can obtain analytic results by diagonalizing $M^{\chi}$ perturbatively ${ }^{8}$ in powers of $v / M_{\text {SUSY }}$. For complex $M_{1,2}$ and $\mu$, one finds to leading order

$$
\begin{aligned}
& Z_{11}^{\chi}=e^{-\frac{i}{2} \phi_{M_{1}}}+O\left(v^{2} / M_{\mathrm{SUSY}}^{2}\right) \\
& Z_{21}^{\chi}=O\left(v^{2} / M_{\mathrm{SUSY}}^{2}\right) \\
& Z_{31}^{\chi}=-\frac{e^{-\frac{i}{2} \phi_{M_{1}}}}{\sqrt{2}} \frac{g_{1} v}{\left|M_{1}\right|^{2}-|\mu|^{2}}\left(M_{1} c_{\beta}+\mu^{*} s_{\beta}\right)+O\left(v^{2} / M_{\mathrm{SUSY}}^{2}\right), \\
& Z_{41}^{\chi}=\frac{e^{-\frac{i}{2} \phi_{M_{1}}}}{\sqrt{2}} \frac{g_{1} v}{\left|M_{1}\right|^{2}-|\mu|^{2}}\left(M_{1} s_{\beta}+\mu^{*} c_{\beta}\right)+O\left(v^{2} / M_{\mathrm{SUSY}}^{2}\right),
\end{aligned}
$$

\footnotetext{
${ }^{6}$ We have $i=1,2,3$ for generation indices and $s=1, \ldots, 6$ for squark mass eigenstates. To recover the expressions in [105], one needs to make the identification $C_{u_{1}}^{\tilde{q}} \leftrightarrow \alpha_{3_{1}}$ etc.

${ }^{7}$ In principle, the $C P$-odd Higgs can contribute to SI $\chi$-quark scattering if $\mu$ or the Yukawa couplings $Y_{q_{i}}$ are allowed to be complex. We exclude this possibility in our numerical analysis since we take real $\mu$ and the real part of $Y_{q_{i}}$ after threshold corrections are included.

${ }^{8}$ For real $M_{1,2}$ and $\mu$, the exact diagonalization of $M^{\chi}$ is known [111-113], although the resulting formulae are not simple.
} 
where $\phi_{M_{1}}$ is the phase of $M_{1}$. Note that the presence of a pole at $\left|M_{1}\right|=|\mu|$ has no physical meaning: it is a consequence of the fact that we assume a bino-like LSP and used non-degenerate perturbation theory to diagonalize the neutralino mass matrix.

In appendix B, we show how (2.23) can be used to simplify (2.21) and (2.22) if flavorviolating effects are neglected, ${ }^{9}$ while allowing for non-universal $A$-terms and squark masses. The resulting expressions read

$$
\begin{aligned}
C_{u_{i}}^{\tilde{q}} & =\frac{g_{1}^{2}}{8}\left[\frac{2}{9} X_{u_{i}} L_{u_{i}}^{+} R_{u_{i}}^{+}+\frac{1}{6} \frac{M_{1}+\mu \cot \beta}{M_{1}^{2}-\mu^{2}}\left(L_{u_{i}}^{+}-4 R_{u_{i}}^{+}\right)\right]+\left(L^{+}, R^{+}\right) \leftrightarrow\left(L^{-}, R^{-}\right), \quad(2.24) \\
C_{d_{i}}^{\tilde{q}} & =-\frac{g_{1}^{2}}{8}\left[\frac{1}{9} X_{d_{i}} L_{d_{i}}^{+} R_{d_{i}}^{+}+\frac{1}{6} \frac{M_{1}+\mu \tan \beta}{M_{1}^{2}-\mu^{2}}\left(L_{d_{i}}^{+}+2 R_{d_{i}}^{+}\right)\right]+\left(L^{+}, R^{+}\right) \leftrightarrow\left(L^{-}, R^{-}\right), \\
C_{u_{i}}^{h, H} & =\frac{g_{1}^{2}}{4} \frac{1}{M_{1}^{2}-\mu^{2}}\left[\left(M_{1}+\mu \cot \beta\right)\left(\frac{c_{\alpha}^{2}}{m_{h}^{2}}+\frac{s_{\alpha}^{2}}{m_{H}^{2}}\right)-\left(M_{1} \cot \beta+\mu\right) s_{\alpha} c_{\alpha}\left(\frac{1}{m_{h}^{2}}-\frac{1}{m_{H}^{2}}\right)\right], \\
C_{d_{i}}^{h, H} & =\frac{g_{1}^{2}}{4} \frac{1}{M_{1}^{2}-\mu^{2}}\left[\left(M_{1}+\mu \tan \beta\right)\left(\frac{s_{\alpha}^{2}}{m_{h}^{2}}+\frac{c_{\alpha}^{2}}{m_{H}^{2}}\right)-\left(M_{1} \tan \beta+\mu\right) s_{\alpha} c_{\alpha}\left(\frac{1}{m_{h}^{2}}-\frac{1}{m_{H}^{2}}\right)\right],
\end{aligned}
$$

where the squark mixing is defined as

$$
X_{u_{i}} \equiv A_{u}^{i i}+\mu \cot \beta \quad \text { and } \quad X_{d_{i}} \equiv A_{d}^{i i}+\mu \tan \beta,
$$

while the squark propagators are

$$
S_{q_{i}}^{ \pm}=\frac{1}{\left(m_{\chi} \pm m_{q_{i}}\right)^{2}-m_{\tilde{q}_{i}^{S}}^{2}+i \epsilon} \quad \text { for } S=L \text { or } R
$$

and $m_{\tilde{q}_{i}^{L}}^{2}$ and $m_{\tilde{q}_{i}^{R}}^{2}$ are the upper and lower diagonal components of the squark (mass) ${ }^{2}$ matrices in (2.18). In deriving (2.26)-(2.27), we have imposed $C P$ conservation so that the neutralino mass parameters $M_{1,2}$ and $\mu$ are real. We also take $0<\beta<\pi / 2$ and $M_{1,2}>0$ so that both signs of $\mu$ are allowed. Expressions for $C_{q_{i}}$ in the $C P$-violating case are provided in appendix B.

Note that:

1. The simplified expressions in (2.24)-(2.25) are valid provided the squarks are sufficiently heavy, i.e. if $m_{q}^{2}+m_{\chi}^{2} \ll m_{\tilde{q}}^{2}$. This requirement is not met for light thirdgeneration squarks, and thus (2.24)-(2.25) must be corrected to account for the oneloop result [72]. To do so, we follow the prescription adopted in [102] and replace all tree-level squark propagators

$$
S_{q_{i}}^{ \pm} \rightarrow-K\left( \pm, m_{q_{i}}, m_{\tilde{q}_{i}}^{S}, m_{\chi}\right)
$$

\footnotetext{
${ }^{9}$ Flavor violation in DM direct detection is strongly suppressed since the effect can only enter via double flavor changes $f \rightarrow j \rightarrow f$ which are experimentally known to be small. Furthermore, the effect of flavor off-diagonal entries can be largely absorbed by a change of the physical squark masses.
} 
in terms of a linear combination $K$ of one-loop functions $I_{i}\left(m_{\chi}, m_{q}, m_{\tilde{q}}\right){ }^{10}$

$$
K\left(\alpha, m_{q}, m_{\tilde{q}}, m_{\chi}\right)=\frac{3}{2} m_{q}\left[m_{q}\left(I_{1}-\frac{2}{3} m_{\chi}^{2} I_{3}\right)-\alpha m_{\chi}\left(I_{2}-\frac{1}{3} I_{5}-\frac{2}{3} m_{\chi}^{2} I_{4}\right)\right],
$$

whose form is given in appendix B of [72]. In the heavy squark limit, the function $K$ agrees with $S_{q_{i}}^{ \pm}$to leading order in $m_{\tilde{q}}^{-2}$.

2. We have made use of the tree-level relation $Y_{q_{i}}=\bar{m}_{q_{i}} / v_{q}$ in order to obtain (2.24)(2.27). For down quarks, however, this relation can be modified by one-loop graphs which induce an effective coupling between $d_{i}$ and the neutral component of $H_{u}$. These corrections $[109,110,114-116]$ are non-decoupling and enhanced by a factor of $\tan \beta .^{11}$ For example, the gluino contribution at one-loop modifies the tree-level relation so that

$$
Y_{d_{i}}=\frac{\bar{m}_{d_{i}}}{v_{d}\left(1+\epsilon_{i} \tan \beta\right)},
$$

where $\epsilon_{i} \simeq-\frac{2 \alpha_{s}}{3 \pi} m_{\tilde{g}} \mu^{*} C_{0}\left(m_{\tilde{g}}^{2}, m_{\tilde{d}_{i}^{L}}^{2}, m_{\tilde{d}_{i}^{R}}^{2}\right)$ and

$$
C_{0}\left(a^{2}, b^{2}, c^{2}\right)=\frac{b^{2}}{\left(a^{2}-b^{2}\right)\left(c^{2}-b^{2}\right)} \log \frac{a^{2}}{b^{2}}+\frac{c^{2}}{\left(a^{2}-c^{2}\right)\left(b^{2}-c^{2}\right)} \log \frac{a^{2}}{c^{2}} .
$$

Since $\bar{m}_{q_{i}} C_{q_{i}}$ is proportional to $Y_{q_{i}} v_{q}$, we can account for (2.32) by a simple rescaling of the Wilson coefficients

$$
C_{d_{i}}^{\tilde{q}, H} \rightarrow \frac{v_{d} Y_{d_{i}}}{\bar{m}_{d_{i}}} C_{d_{i}}^{\tilde{q}, H},
$$

where we include corrections $[109,110,114-116]$ beyond the gluino loop (2.32). These threshold corrections feature in our analysis of heavy Higgs $H$ and sbottom contributions (section 3) to the SI amplitude. Note that corrections to the light Higgs coupling $h \bar{d} d$ cancel in the relation $\bar{m}_{u_{i}}=Y_{u_{i}} v_{u}$.

\section{Simplified models: blind spots and isospin violation}

We now apply our analytic results (2.24)-(2.27) to four simplified models; each motivated by the following experimental and naturalness considerations. Firstly, the ATLAS [106] and CMS [107] experiments at the LHC have discovered a Higgs boson with SM-like properties and a mass below the upper bound $\lesssim 135 \mathrm{GeV}$ of the MSSM. Secondly, a natural resolution of the gauge hierarchy problem requires several conditions $[46,52,53]$ to be met:

- In order to cancel the top-quark correction to the Higgs mass parameter $m_{H_{u}}^{2}$, top squarks must be light with masses in the sub-TeV range;

\footnotetext{
${ }^{10}$ The term proportional to $I_{3}$ in (A5) of [102] is missing a factor of $m_{q}$.

${ }^{11}$ In principle, large $A$-terms can also change the values of $Y_{q_{i}}$ significantly [110, 117-120]. However, this effect drops out in the Higgs-quark-quark couplings where the effective (physical) mass enters. Furthermore, since we assume flavor-universal $A$-terms in our numerical analysis, the effect cannot be very large without violating vacuum stability bounds.
} 


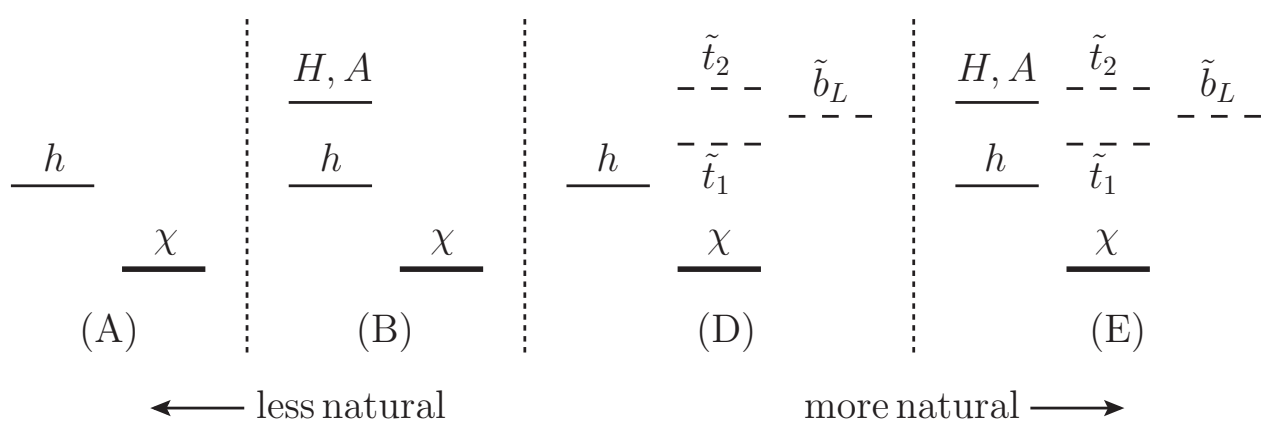

Figure 3. Spectra of the simplified models (A-D) considered in this work. For each model, the LSP $\chi$ is assumed to be bino-like and may be accompanied by a nearly degenerate tau slepton in order to produce the observed DM relic density. The SM-like Higgs is denoted by $h$, while all other states are assumed to lie below $1 \mathrm{TeV}$, including Higgsinos (not shown). From left-to-right, the spectra become increasingly more natural as one includes the additional Higgs states $H, A$ and third-generation squarks $\tilde{t}_{1}, \tilde{t}_{2}, \tilde{b}_{L}$. In general, $\chi$ may be heavier than $h, H$, and $A$.

- The gluino mass must be around a $\mathrm{TeV}$ in order to prevent radiative corrections driving the stop masses too heavy;

- Light Higgsinos must be present in the spectrum so that tree-level electroweak symmetry breaking implies that $\mu \sim v$ is satisfied.

It has also been observed [121] that naturalness constrains the additional Higgs bosons $H, H^{ \pm}, A$ to not be too heavy. Barring the gluino, the current experimental bounds on the masses of the above particles are rather weak. In contrast, the mass of the gluino and squarks of the first two generations are constrained to lie above $1 \mathrm{TeV}$. Therefore, naturalness prompts us to consider the simplified models shown in figure 3, where we start from a minimal, light particle spectrum necessary to have bino-like DM scattering [model (A)] and successively include as active degrees of freedom those particles which are (a) required to be light by naturalness, and (b) relevant for DM direct detection. Note that due to $\mathrm{SU}(2)_{L}$ invariance, the models (C-D) involving two light stops always require a light sbottom in the spectrum. (Only if there is a single, mostly right-handed stop, can sbottoms be decoupled.)

In general, a bino-like LSP produces a DM relic density that is too large in most of the parameter space considered in section 3. However, the overproduction of bino-like DM in the MSSM can be diluted by either $s$-channel resonance exchange involving $Z, h, H, A$, or $\chi$ - $\tilde{f}$ co-annihilation with a sfermion $\tilde{f}$ that is nearly degenerate in mass with $\chi{ }^{12}$ Both mechanisms [122] increase the annihilation cross section before thermal freeze-out and can produce the observed relic abundance. In each of the models shown in figure 3, the relic density constraint may be satisfied by either mechanism or, if necessary, by extending the spectrum to include a tau slepton $\tilde{\tau}$ which generates additional co-annihilations $[123,124]$. Since the $\tilde{\tau}$ mass can be tuned without affecting naturalness or DM direct detection, we do not consider the DM relic density constraint in our subsequent analysis.

\footnotetext{
${ }^{12}$ See e.g. [17] for a detailed analysis of these effects in the pMSSM.
} 
Similarly, we do not consider the constraint from the anomalous magnetic moment of the muon $a_{\mu}$, whose world average is dominated by the Brookhaven measurement [125]. The resulting value deviates from the SM prediction by $3-4 \sigma$, depending on the details of the evaluation of the hadronic contributions [126, 127]. Recent developments in the evaluation of the SM prediction include: the QED calculation has been carried out at 5-loop accuracy [128], after the Higgs discovery [106, 107] the electroweak contribution is complete at two-loop order $[129,130]$, and hadronic corrections have been considered at third order in the fine-structure constant $[131,132]$. Although an improved determination of the leading hadronic contribution, hadronic vacuum polarization, mainly requires improved data input, see [126, 133-135], the uncertainties in the subleading hadronic-light-by-light contribution have been notoriously difficult to estimate due to substantial model dependence [126, 127, 136]. Recently, data-driven techniques have been put forward to reduce the model dependence based on dispersion relations [137-140], and a first lattice calculation has become available [141]. All these efforts are motivated by two new experiments, at FNAL [142] and J-PARC [143], which each aim at improving the measurement by a factor of 4 and thus help clarify the origin of the discrepancy between experiment and the SM prediction.

Should the discrepancy persist, an explanation within the MSSM is possible provided certain assumptions are made about the SUSY parameters entering the smuon, chargino, and neutralino mass matrices. If these parameters are all equal to $M_{\text {SUSY }}$, then a positive contribution to $a_{\mu}$ requires $\operatorname{sign}\left(\mu M_{2}\right)>0$ since the dominant one-loop amplitude scales approximately with $\mu M_{2} \tan \beta / M_{\text {SUSY }}^{2}$; see e.g. the review [144] and references therein. In the blind spot regions where $\mu<0$, this condition would require us to relax the assumption that $M_{2}>0$. However, the requirement $\operatorname{sign}\left(\mu M_{2}\right)>0$ does not necessarily apply if the SUSY mass parameters are non-degenerate. For example, it has been shown [145-147] that a positive contribution to $a_{\mu}$ can arise if $\left|M_{1}\right|, m_{\tilde{\mu}_{R}} \ll\left|M_{2}\right|, m_{\tilde{\mu}_{L}}$, in which case $\mu$ and $M_{1,2}$ must have opposite sign. The key point is that neither the sign of $M_{2}$ nor the smuon masses are relevant for our analysis of SI scattering, so it would be possible to account for the experimental value of $a_{\mu}$ by a suitable choice of these parameters. Furthermore, the discrepancy could also be explained by large $A_{\mu}$ terms $[118,148,149]$ not correlated with DM scattering.

We conclude this section by anticipating a key result of our analysis: isospin-violating effects can be magnified in the proximity of blind spots, where the SI direct-detection cross section lies below the lower bounds set by the irreducible neutrino background. For these parameter-space configurations, the SI amplitude itself becomes tiny and hence more susceptible to small variations in the input quantities, such as corrections from isospin breaking. In particular, the ratio of proton and neutron SI cross sections becomes very sensitive to the values of the scalar matrix elements and their uncertainties $\delta f_{n, p}$,

$$
\left(\delta \frac{f_{n}}{f_{p}}\right)^{2}=\left(\frac{\delta f_{n}}{f_{p}}\right)^{2}+\left(\frac{f_{n} \delta f_{p}}{f_{p}^{2}}\right)^{2}
$$

so that the overall uncertainty on $f_{n} / f_{p}$ can become large near blind spots where $f_{p} \simeq 0$. In each of the four simplified models (A-D), we examine the amount of isospin violation associated with the three methods of section 2.1. 

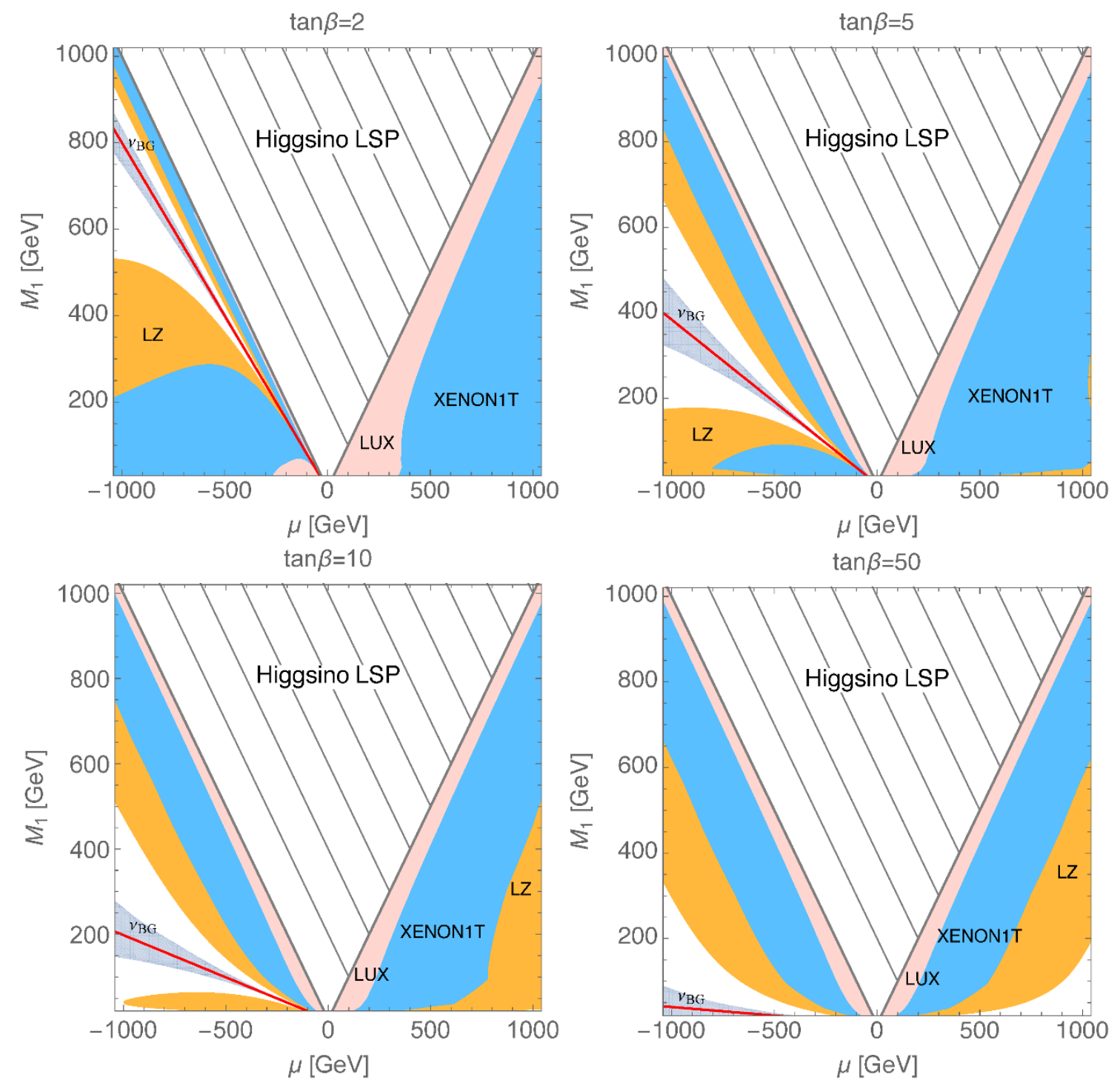

Figure 4. Current and projected limits on SI $\chi$-xenon scattering due to $h$ exchange with $\tan \beta=2$ (top left), 5 (top right), 10 (bottom left), and 50 (bottom right). The pink band shows the existing constraints from LUX [21], while projected limits from XENON1T [22] and LZ [23] are given by the blue and orange regions respectively. The blind spot where the SI cross section vanishes is denoted by the red line and lies within the irreducible neutrino background $\left(\nu_{\mathrm{BG}}\right)$ shown in gray. We assume the LSP is bino-like (figure 3), so do not consider the triangular, hatched region where $\mu<M_{1}$ and $\chi$ becomes Higgsino-like.

\subsection{SM-like Higgs exchange}

We begin by considering the minimal particle spectrum for which an observable SI cross section is possible. From the rightmost diagram in figure 2, it seems reasonable to conclude that the SM-like Higgs and the bino-like LSP is sufficient in this case. However, in the 
limit $m_{A} \gg m_{Z},(2.26)$ and (2.27) become

$$
C_{u_{i}}^{h}=C_{d_{i}}^{h}=\frac{g_{1}^{2}}{4 m_{h}^{2}} \frac{1}{M_{1}^{2}-\mu^{2}}\left(M_{1}+\mu s_{2 \beta}\right), \quad u_{i}=u, c, t, \quad d_{i}=d, s, b,
$$

and thus the scattering amplitude decouples with the Higgsino mass $\mu$. It follows that a measurable cross section due to Higgs exchange implies the presence of light Higgsinos in the spectrum, thereby satisfying one of the minimal naturalness requirements. Although this feature does not prevent the reintroduction of fine-tuning in the MSSM altogether, it becomes relevant in our subsequent analysis where light stops are added to the spectrum.

To compare (3.2) to data, we first note that $C_{q_{i}}^{h}$ vanishes when

$$
M_{1}+\mu s_{2 \beta}=0,
$$

and thus a blind spot arises in the SI cross section provided $\mu$ is negative. The prospects for constraining this feature (3.3) have been extensively analyzed [30] for $\chi$-nucleon scattering. To examine isospin violation, however, we need limits on $\chi$-nucleus cross sections, so we use (2.11) in order to constrain the relevant parameter space.

Let us first consider the limits associated with (3.2) when the scalar matrix elements $f_{q}^{N}$ of Method 1 are employed. In figure 4, we update the results from [30] and show constraints for various values of $\tan \beta$ in the $\left(\mu, M_{1}\right)$ plane from current and upcoming xenon experiments. For $\mu>0$, we find that only a narrow strip is excluded by the existing limits from LUX [21], while the projected reach from XENON1T [22] and LZ [23] will probe most of the naturalness-preferred region where $\mu$ is of order $v$. As $\tan \beta$ is increased, the term $\propto \mu$ in $C_{q_{i}}^{h}$ is suppressed, thereby weakening the direct detection limits. If no signal is seen at LZ, then the allowed parameter space is focused towards $\tan \beta=50$ and values of $M_{1} \lesssim 200 \mathrm{GeV}$. In the $\mu<0$ region and for small $\tan \beta$, the naturalness-preferred values of $\mu$ occur at $|\mu| \simeq M_{1}$ and are concentrated near the blind spot. Although the irreducible neutrino background make this region difficult to probe experimentally, larger values of $\tan \beta$ decrease the blind spot slope, so that natural values of $\mu$ become allowed for $M_{1} \lesssim 300-400 \mathrm{GeV}$.

By taking a slice through the $\left(\mu, M_{1}\right)$ plane, we can also extract the limits due to a small mass splitting $|\mu|-M_{1}=80 \mathrm{GeV}$ between the bino and Higgsinos. This choice is motivated by the current CMS results [150] on same-flavor opposite-sign dilepton searches. Here CMS sees a $2.6 \sigma$ deviation which can be explained by a heavier neutralino decaying to a lighter one. Figure 5 shows the resulting constraints, where we plot the SI cross sections as a function of the bino mass. For $\mu>0$, the limits from LUX are stringent, with values below $M_{1} \simeq 600 \mathrm{GeV}$ excluded. The strength of these limits is due to an enhancement in the amplitude (3.2) from both a nearly degenerate denominator and lack of interference in the numerator terms. For $\mu<0$, there are no constraints from LUX, although XENON1T and LZ will exclude the whole parameter space in the absence of a DM signal.

We now examine the hadronic uncertainties associated with each of the three methods discussed in section 2.1. For $h$ exchange, the Wilson coefficient (3.2) is independent of quark flavor, so the SI amplitude (2.2) factorizes

$$
\frac{f_{N}}{m_{N}}=C_{q_{i}}^{h}\left(\frac{2}{9}+\frac{7}{9} \sum_{q=u, d, s} f_{q}^{N}\right) .
$$




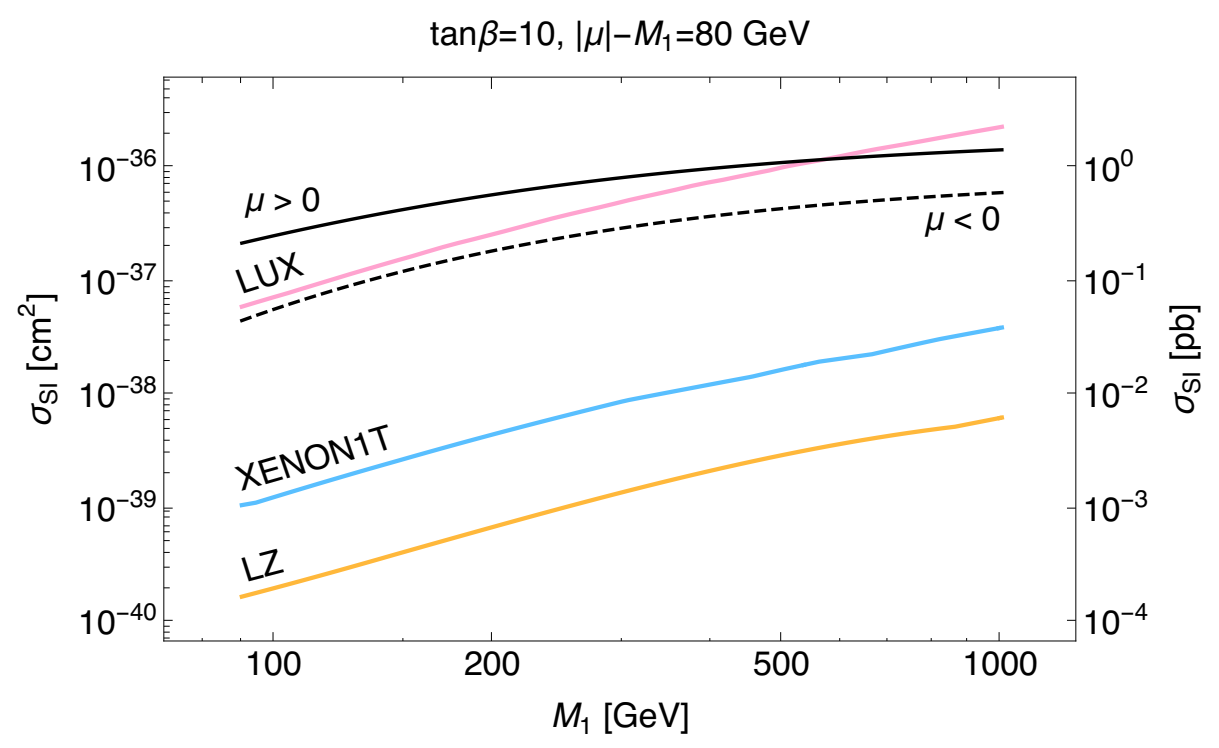

Figure 5. SI $\chi$-xenon cross sections for $h$ exchange with $\tan \beta=10$ and a small mass difference $|\mu|-M_{1}=80 \mathrm{GeV}$ between the Higgsino and bino. The solid (dashed) black line corresponds to $\mu>0(\mu<0)$. Shown are the limits from LUX [21], XENON1T [22], and LZ [23], with the same color coding as in figure 4 .

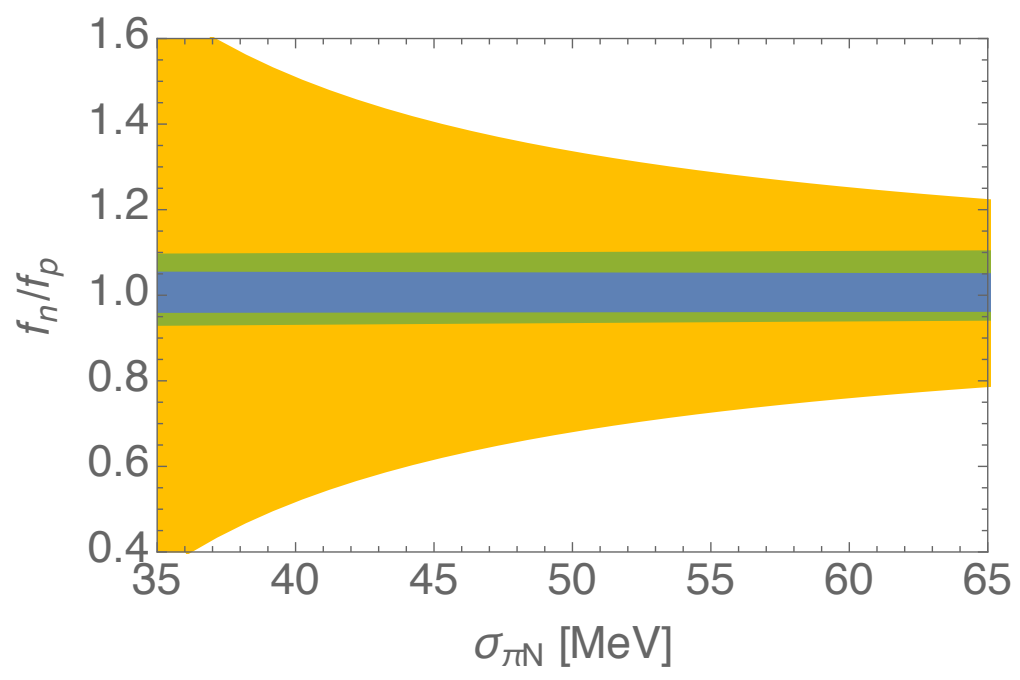

Figure 6. Amount of isospin violation in terms of $f_{n} / f_{p}$ due to $h$ exchange in $\chi$-nucleus scattering. The colored bands correspond to the $1 \sigma$ uncertainties associated with the different determinations of the scalar matrix elements $f_{q}^{N}$ discussed in section 2.1. The blue band corresponds to Method 1, while the orange and green bands correspond to Method 2 and Method 3 respectively. 
Evidently, the resulting SI cross section is sensitive to the value of $f_{s}^{N}$, with a dramatic effect observed [99] on the regions of excluded parameter space when the typically large value $f_{s}^{N} \approx 0.25$ of Method 3 is replaced with much smaller determinations (2.13) from the lattice. We emphasize that this sensitivity is also present in any analysis of isospin violation, where $f_{n} / f_{p}$ is the quantity of interest. For the present discussion, (3.4) implies that the ratio

$$
\frac{f_{n}}{f_{p}}=\left(\frac{m_{n}}{m_{p}}\right) \frac{2+7 \sum f_{q}^{n}}{2+7 \sum f_{q}^{p}}
$$

is independent of $C_{q_{i}}^{h}$, and thus isospin violation is entirely determined by hadronic quantities. In figure 6 we compare the uncertainties on $f_{n} / f_{p}$ as a function of $\sigma_{\pi N}$. For Methods 1 and 3, we find stability across a large range of $\sigma_{\pi N}$ values, with isospin violation allowed at around the five and ten percent level respectively. As noted in [151], this stability is due to the fact that the constant term of $\frac{2}{9}$ in (3.4) dominates the remainder whenever $f_{s}^{N}$ is fixed by lattice input. In contrast, the $\chi \mathrm{PT}_{3}$ formalism of Method 2 produces a strong dependence of $f_{s}^{N}$ on $\sigma_{\pi N}$, which in turn affects $f_{n} / f_{p}$. From figure 6 , isospin violation greater than $50 \%$ is allowed, in marked contrast to the precision of Method 1. This example clearly demonstrates the huge uncertainties associated with Method 2, which, however, is still used in the literature $[10,152]$.

\subsection{Light and heavy Higgs exchange}

Let us now extend model (A) to include the heavy Higgs bosons $H, A, H^{ \pm}$[model (B) in figure 3]. The inclusion of these additional degrees of freedom is motivated by naturalness [121], however, only $H$ contributes to the SI cross section (figure 2).

From our simplified expressions (2.26)-(2.27), we see that the couplings to up and down quarks differ by a factor of $\tan \beta$, but are identical ${ }^{13}$ among different generations $i=1,2,3$. As a result, the SI amplitude may be expressed as

$$
\frac{f_{N}}{m_{N}}=C_{u_{i}}^{h, H} U_{N}+C_{d_{i}}^{h, H} D_{N},
$$

where

$$
U_{N}=f_{u}^{N}+2 f_{Q}^{N} \quad \text { and } \quad D_{N}=f_{d}^{N}+f_{s}^{N}+f_{Q}
$$

collect the scalar coefficients associated with the up- and down-type Wilson coefficients. A blind spot occurs if the condition

$$
C_{u_{i}}^{h, H} U_{N}+C_{d_{i}}^{h, H} D_{N} \simeq 0
$$

is satisfied, and the resulting suppression of the SI cross section has been identified numerically [39, 55-57] and further studied analytically [38]. In the latter case, an explicit formula [38] for the blind spot can be found for moderate to large values of $\tan \beta$ and $m_{A}>m_{h}$ :

$$
\frac{2}{m_{h}^{2}}\left(M_{1}+\mu s_{2 \beta}\right)+\mu \tan \beta \frac{1}{m_{H}^{2}} \simeq 0 .
$$

\footnotetext{
${ }^{13} \mathrm{Up}$ to threshold corrections (2.34), which enhance $C_{d_{i}}^{H}$ by tens of percent at large $\tan \beta$. Their inclusion does not have a large impact on the numerical analysis.
} 


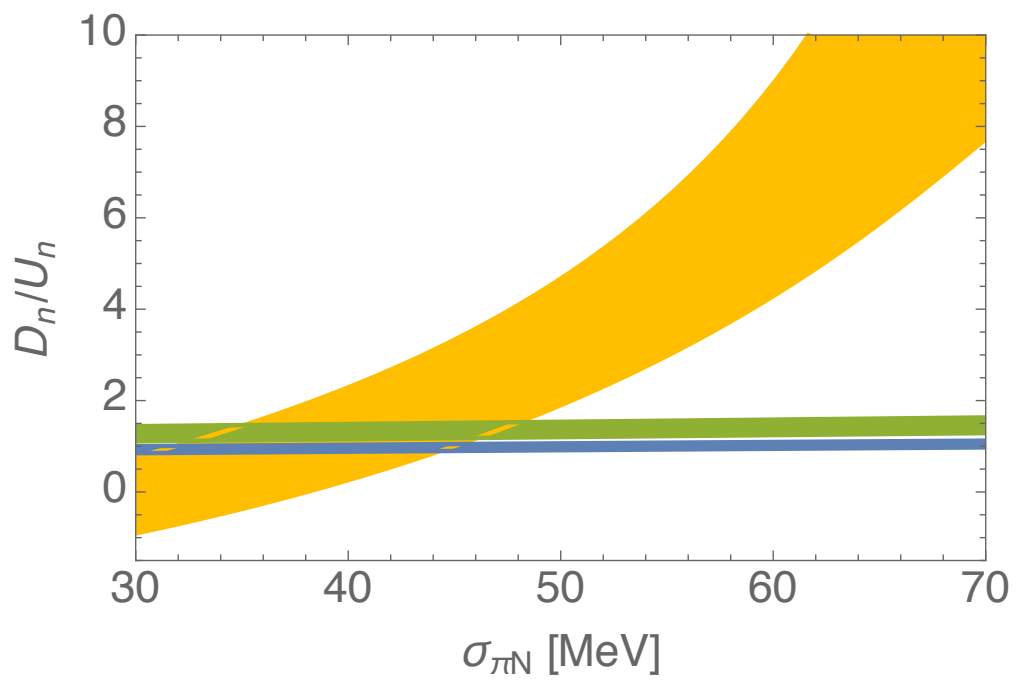

Figure 7. Dependence on $\sigma_{\pi N}$ in the ratio of the hadronic terms $U_{N}$ and $D_{N}$ pre-multiplying the up- and down-type Wilson coefficient for $h, H$ exchange (3.6). Shown is the case for $N=$ neutron, with similar results obtained for $N=$ proton. Color coding as in figure 6.

In effect, (3.8) has been recast as an interference condition between the $h$ and $H$ amplitudes; a feature which has important consequences for isospin violation in the MSSM. As with $h$ exchange, negative values of $\mu$ are required in order to generate the blind spot. However, note that in the vicinity of (3.3), the first term in (3.9) is suppressed, so in some cases the contribution from $H$ exchange may dominate the scattering amplitude [38].

A crucial step in the derivation [38] of (3.9) is the observation that $U_{N} \approx D_{N}$ numerically. Deviations of $D_{N} / U_{N}$ from unity have the effect of shifting the location of the blind spot (3.9), so it is necessary to determine this ratio precisely. In figure 7 , we display the sensitivity of $D_{N} / U_{N}$ to $\sigma_{\pi N}$ for each of the three methods of section 2.1. Similar to our analysis of $h$ exchange (figure 6), we find that Methods 1 and 3 are stable across a large range of $\sigma_{\pi N}$ values, with $D_{N} / U_{N} \simeq 1$ tightly constrained. In contrast, Method 2 exhibits a strong dependence on $\sigma_{\pi N}$ and for $\sigma_{\pi N} \gtrsim 45 \mathrm{MeV}$, the location of the blind spot (3.9) can get shifted by a factor of eight or more. These findings illustrate again the importance of using a well-controlled framework for the hadronic input quantities.

Let us now examine the experimental limits associated with $\chi$-xenon scattering. In figure 8 , we show constraints in the $\left(m_{A}, \tan \beta\right)$ plane for two benchmark values of $M_{1}$ and $\mu$. We find that as the mass splitting between $M_{1}$ and $\mu$ is decreased, the limits become significantly stronger. This is because the amplitude $C_{q_{i}}^{h, H}$ scales like $\sim 1 /\left(M_{1}^{2}-\mu^{2}\right)$, so the naturalness requirement of light Higgsinos implies strong constraints on the SI cross section. We also find blind spots similar to those previously identified [38, 39, 55-57], and see that the strongest limits are due to $H, A \rightarrow \tau^{+} \tau^{-}$searches [153] as one approaches (3.9) from below.

What about isospin violation in this model? Unlike single $h$ exchange, where $f_{n} / f_{p}$ is entirely fixed (3.5) by hadronic quantities, the blind spot (3.9) for light and heavy Higgs bosons involves destructive interference between the respective amplitudes. In general, we find that isospin violation can be enhanced in the vicinity of such blind spots because 

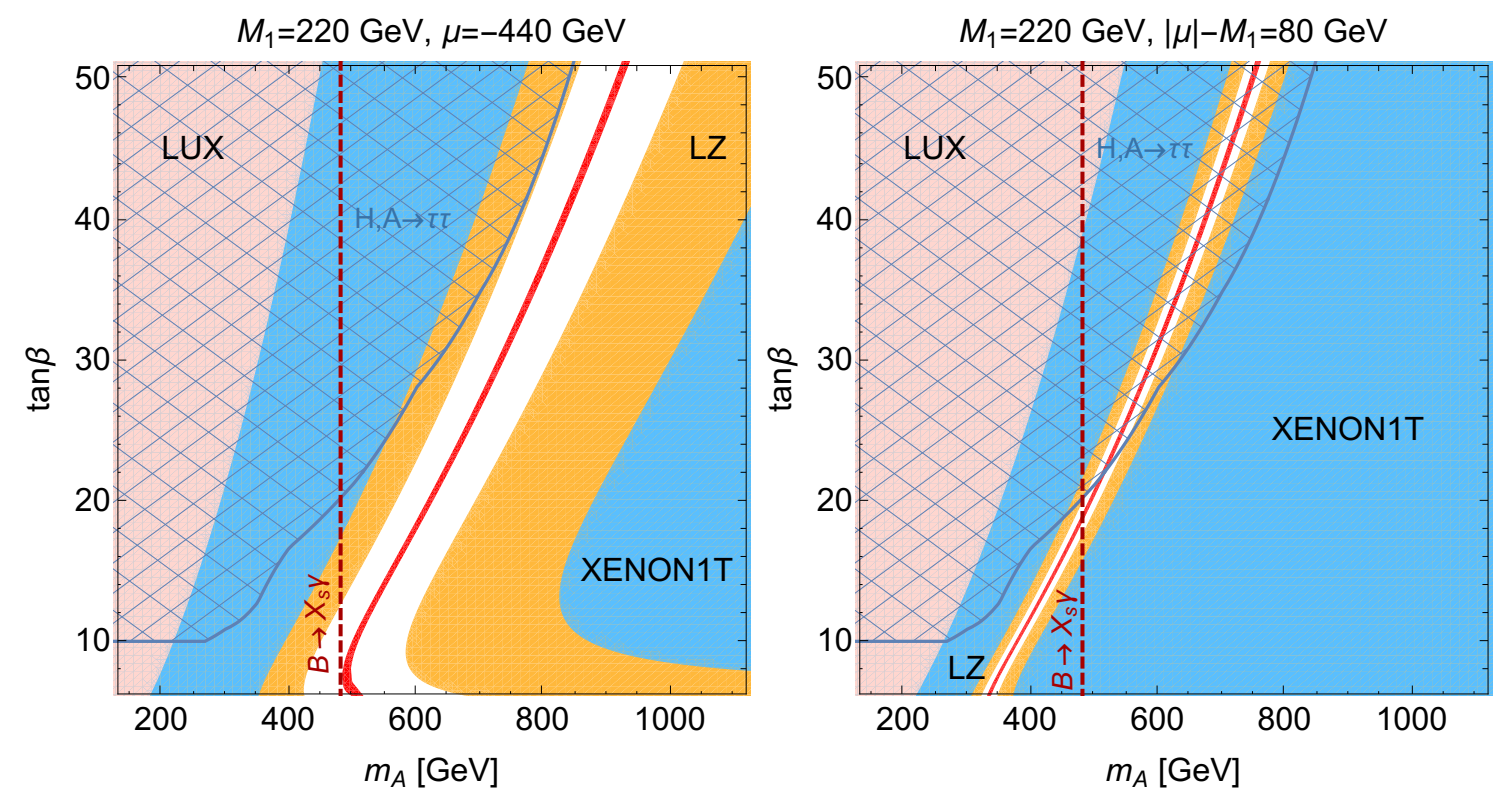

Figure 8. Current and projected limits on SI $\chi$-xenon scattering due to $h, H$ exchange with different benchmark values for $M_{1}$ and $\mu$. Excluded regions and the blind spot are color-coded as in figure 4, with the cross-hatched region in dark-blue corresponding to CMS limits [153] on $H, A \rightarrow \tau^{+} \tau^{-}$. The region to the left of the dark-red dashed line at $m_{A} \simeq m_{H^{+}} \simeq 480 \mathrm{GeV}$ is excluded by $B \rightarrow X_{s} \gamma[154]$.

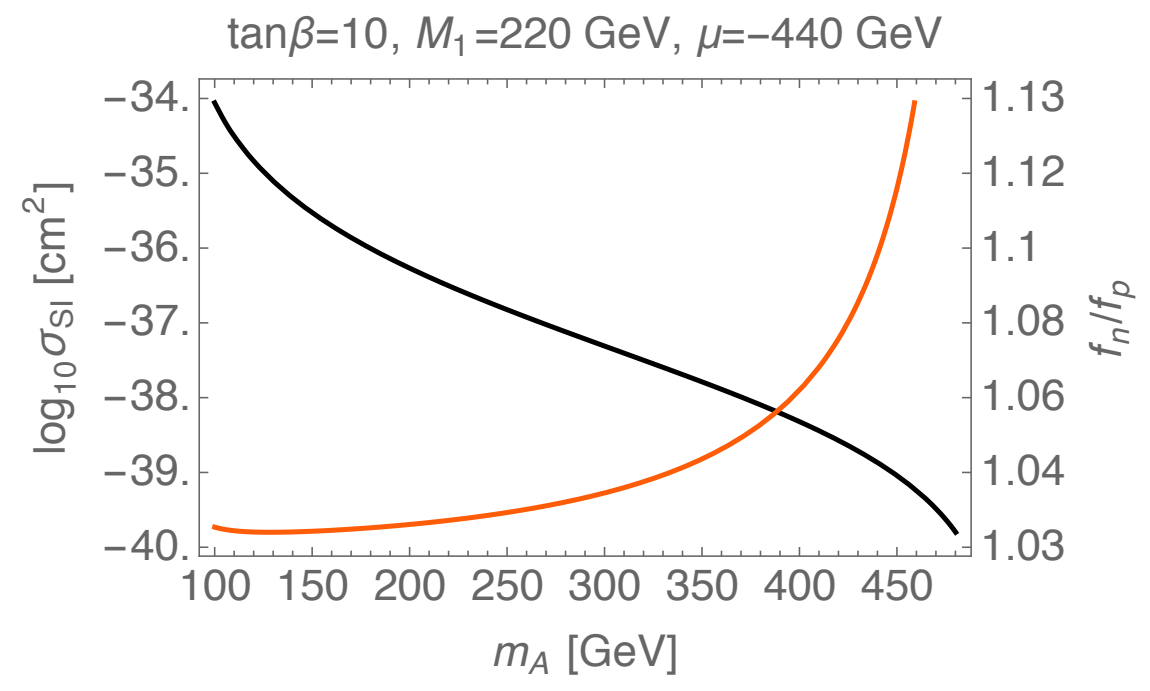

Figure 9. Pseudoscalar Higgs mass $m_{A}$ dependence of the SI $\chi$-xenon cross section (black) and the central value of $f_{n} / f_{p}$ (red) as determined by Method 1 .

$f_{n} / f_{p}$ becomes sensitive to the scalar matrix elements and their uncertainties (3.1). This is evident in figure 9 , where the central value of $f_{n} / f_{p}$ (determined by Method 1 ) reaches $\approx 15 \%$ as the blind spot is approached with increasing $m_{A}$.

In figure 10 we compare the amount of isospin violation allowed by Methods 1-3. As observed in single $h$ exchange, the uncertainties associated with Method 2 are large, and 


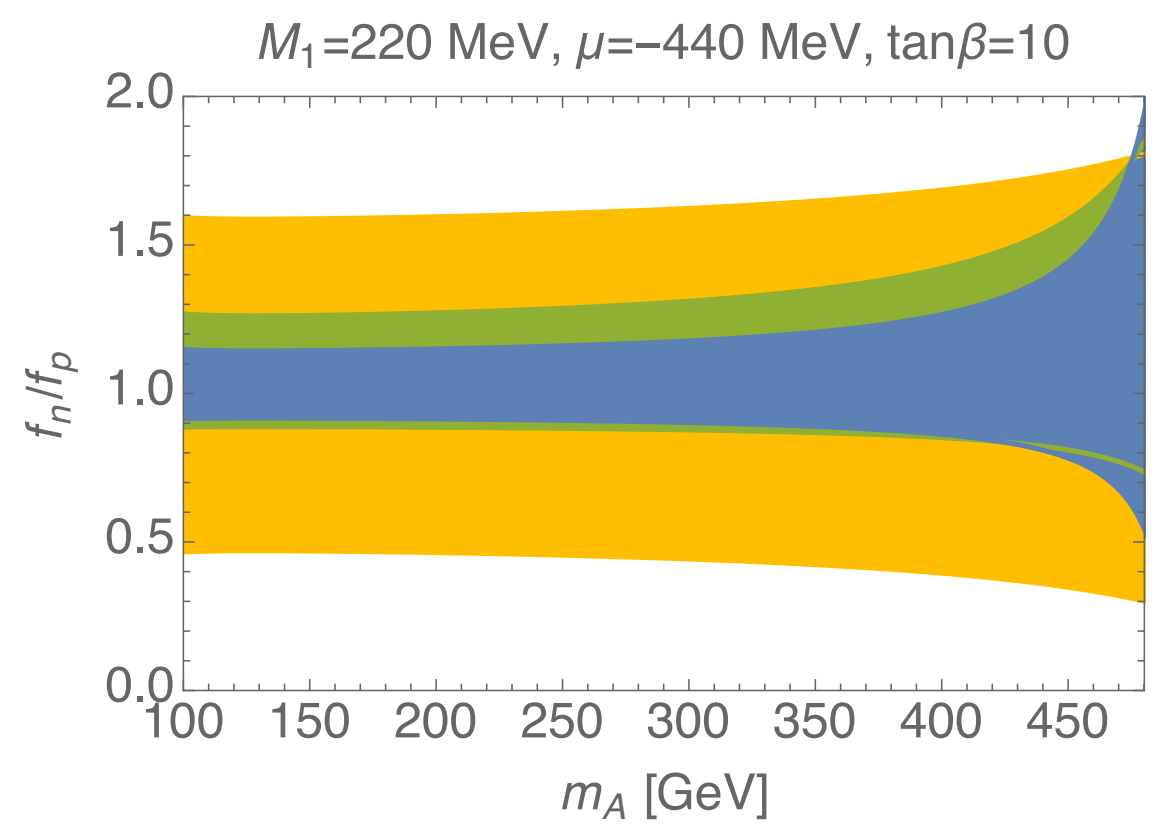

Figure 10. Amount of isospin violation in terms of $f_{n} / f_{p}$ due to $h, H$ exchange in $\chi$-xenon scattering. The shaded regions show the uncertainty on $f_{n} / f_{p}$ due to each determination of the scalar matrix elements listed in section 2.1. Color coding as in figure 6 .

differ by a factor of two or more for $m_{A} \lesssim 400 \mathrm{GeV}$. For $m_{A} \gtrsim 400 \mathrm{GeV}$, a comparison between the Methods is obscured by the fact that the location of the blind spot is shifted depending on deviations from $D_{N}=U_{N}$ (figure 7).

In figure 11 we display the allowed ranges of isospin violation due to Method 1 for two values of $\tan \beta$. For $\tan \beta=10$, the recent limit from $B \rightarrow X_{s} \gamma$ [154] excludes the region below the blind spot at $m_{A} \approx 500 \mathrm{GeV}$. Above the blind spot, the absence of a signal at LZ would imply that isospin violation as large as $10 \%$ becomes allowed. For $\tan \beta=20$, the current limit from LUX [21] allows around $10 \%$ isospin violation, although this occurs at a value of $m_{A} \approx 200 \mathrm{GeV}$ already excluded by the limits from $H, A \rightarrow \tau^{+} \tau^{-}[153]$ and $B \rightarrow X_{s} \gamma$ [154]. The absence of a signal at XENON1T would allow $\approx 20 \%$, while at LZ this would imply that isospin violation as large as $40 \%$ is allowed within the uncertainties as one approaches the blind spot from below. As illustrated in figure 9 , it is important to note that not only the allowed range, but also the central value of $f_{n} / f_{p}$ can increase as the blind spot is approached. In consequence, the absence of signals in SI DM searches pushes the parameter space into blind spots, at which $f_{n} / f_{p}$ may become large and thus the accurate determination of $\delta f_{n}$ and $\delta f_{p}$ becomes paramount. A comparison for other nuclear targets can be inferred by taking the limits on $f_{n} / f_{p}$ and comparing against figure 1 .

\subsection{SM-like Higgs and light squark exchange}

In the previous subsection, we investigated parameter configurations (3.8) where the $h, H$ amplitudes interfere destructively and observed that isospin violation can be enhanced in the proximity of these blind spots (figure 11). Next, we examine if blind spots still exist once third-generation squarks are added to the spectrum of model (A). 

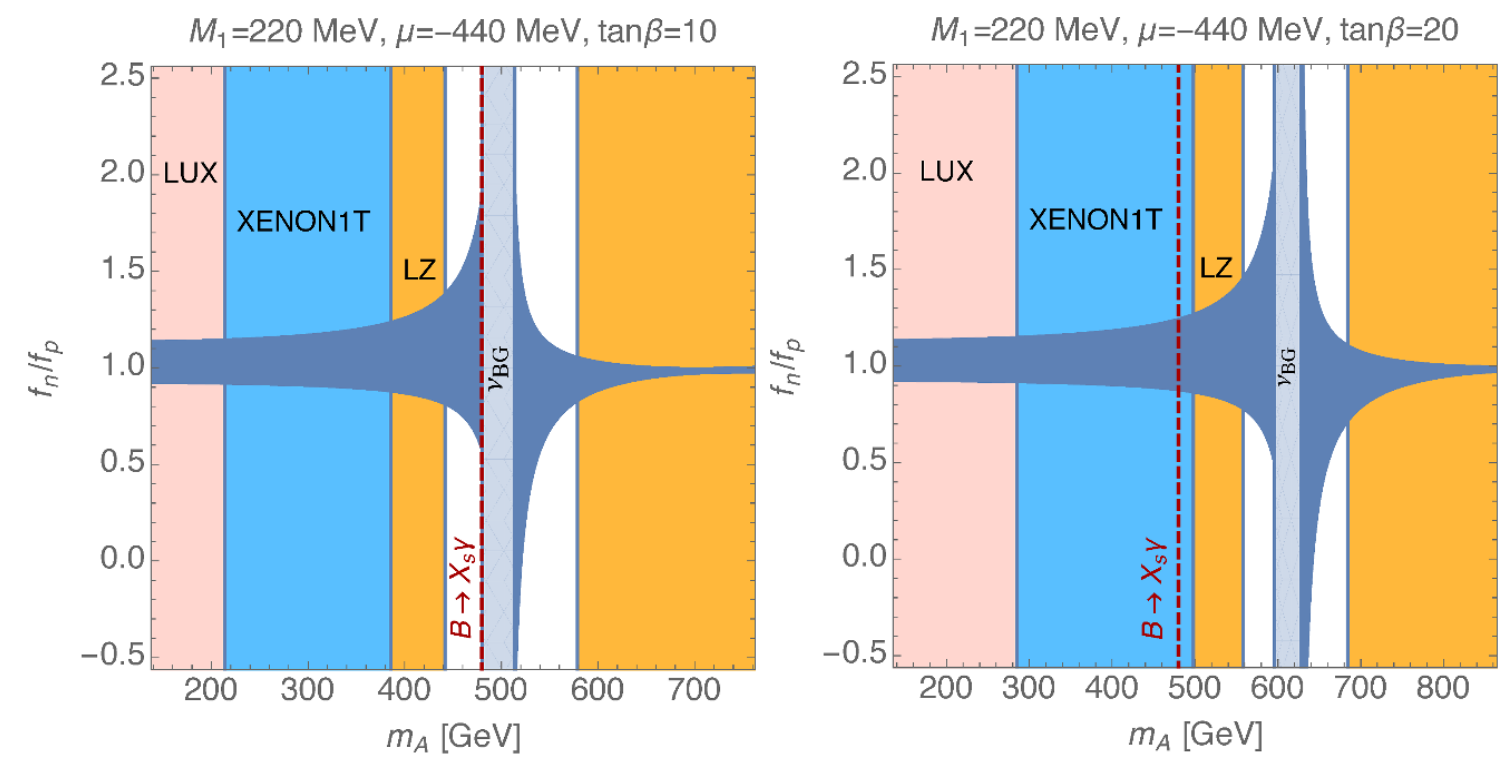

Figure 11. Current and projected results for the measure $f_{n} / f_{p}$ of isospin violation arising from $h, H$ exchange in SI $\chi$-xenon scattering for $\tan \beta=10$ (left) and 20 (right). The dark blue region denotes the uncertainty on $f_{n} / f_{p}$ as determined by Method 1 . The existing and projected experimental limits are color coded as in figure 4 , with the irreducible neutrino background $(\nu \mathrm{BG})$ shown by the central gray band. The region to the left of the dark red dashed line at $m_{A} \simeq 480 \mathrm{GeV}$ is excluded by $B \rightarrow X_{s} \gamma[154]$.

The effects on the SI amplitude due to squarks from the first two generations were considered in [30] (including $h$ exchange) and shown to be small due to the stringent limits from LHC searches. However, the existing limits on third-generation squark masses are much weaker, so that effects from stops and sbottoms can be significantly larger.

The simplest model, i.e. with minimal particle content, would involve a single, mostly right-handed stop $\tilde{t}_{R}$. However, as one can see from (2.24), this contribution is not $\tan \beta$ enhanced and thus the $h$ contribution (3.2) dominates the SI cross section. Therefore, we consider a spectrum where $h$ and $\tilde{t}_{1,2}$ are the dynamical degrees of freedom [model (C) in figure 3]. Since a left-handed stop is always associated with a left-handed sbottom $\tilde{b}_{L}$, the sbottom contribution must be included as well. Although this does not increase the number of free parameters, we can see from (2.25) that the sbottom amplitude is $\tan \beta$ enhanced and, crucially, can compete with the Higgs contribution to the SI amplitude. Note that while the Higgs amplitude vanishes with decoupling Higgsinos (thereby violating the minimal naturalness conditions), this is not the case for the sbottom contribution, which possesses a term proportional to $\mu$.

Using our simplified expressions, we find that a blind spot occurs if the condition

$$
\frac{6}{m_{h}^{2}}\left(M_{1}+\mu s_{2 \beta}\right)\left[\sum_{q} f_{q}^{N}+3 f_{Q}^{N}\right]-\frac{1}{M_{1}^{2}-m_{\tilde{b}_{L}}^{2}}\left(M_{1}+\mu \tan \beta\right) f_{Q}^{N} \simeq 0
$$

is satisfied. Here, we have ignored the numerically small stop contribution $C_{t}^{\tilde{t}}$ and approximated the effects due to sbottom loops $(2.31)$ by the tree-level propagator. This latter 
approximation is for illustrative purposes only, and in our numerical analysis we use the exact one-loop expressions. Using the scalar matrix elements from Method 1, we have $\sum_{q} f_{q}^{N}+3 f_{Q}^{N} \simeq \frac{1}{3}$ and $f_{Q}^{N} \simeq \frac{1}{15}$. Therefore, for moderate to large values of $\tan \beta$ the blind spot condition simplifies to

$$
\frac{30}{m_{h}^{2}}\left(M_{1}+\mu s_{2 \beta}\right)+\mu \tan \beta \frac{1}{m_{\tilde{b}_{L}}^{2}-M_{1}^{2}} \simeq 0 .
$$

As expected, this blind spot shares common features with the one found [38] for $h, H$ exchange (3.9): it requires negative values of $\mu$, so that the couplings to $h$ are suppressed and destructive interference between the $h$ and $\tilde{b}_{L}$ amplitudes can occur. However, larger values of $|\mu|$ are required in order to overcome the factor of 30 in the Higgs amplitude.

Before determining the experimental limits on this model, let us consider the size of the parameter space. The $h$ amplitude depends on $M_{1}, \mu$, and $\tan \beta$, so we need to add the parameters $\left(\mathbf{m}_{Q}\right)_{33},\left(\mathbf{m}_{U}\right)_{33}$, and $X_{t}$ of the stop mass matrix. As noted above, the sbottom contribution does not involve additional parameters since the left-handed sbottom mass is given by

$$
m_{\tilde{b}_{L}}^{2}=\left(\mathbf{m}_{Q}\right)_{33}^{2}+m_{b}^{2}-\left(\frac{1}{2}-\frac{1}{3} s_{W}^{2}\right) m_{Z}^{2} c_{2 \beta} .
$$

To reduce the number of free parameters we fix $\left(\mathbf{m}_{Q}\right)_{33} \approx\left(\mathbf{m}_{U}\right)_{33}$, in which case the leftand right-handed entries in the stop mass matrix become nearly degenerate, while the physical mass eigenvalues read

$$
m_{\tilde{t}_{1}, \tilde{t}_{2}}^{2}=m_{\tilde{t}_{L}}^{2} \mp m_{t} X_{t} .
$$

This allows us to express our simplified expressions (2.24)-(2.25) in terms of the physical masses and compare with collider limits in the $\left(m_{\tilde{t}_{1}}, M_{1}\right)$ plane. In order for light stops to generate the correct Higgs mass in the MSSM, we assume these states are mixed in such a way so as to give a maximal contribution to the Higgs mass. This is achieved by noting that the one-loop stop contribution to the Higgs mass

$$
m_{h}^{2} \approx m_{Z}^{2} c_{2 \beta}^{2}+\frac{3}{4 \pi^{2}} \frac{\bar{m}_{t}^{4}}{v^{2}}\left[\ln \frac{\hat{m}_{\tilde{t}}^{2}}{\bar{m}_{t}^{2}}+\frac{X_{t}^{2}}{\hat{m}_{\tilde{t}}^{2}}\left(1-\frac{X_{t}^{2}}{12 \hat{m}_{\tilde{t}}^{2}}\right)\right]
$$

is maximized at $\left|X_{t}\right|=X_{t}^{\max }=\sqrt{6} \hat{m}_{\tilde{t}}$, where $\hat{m}_{\tilde{t}}^{2}=m_{\tilde{t}_{1}} m_{\tilde{t}_{2}}$ is the average stop mass. Since $m_{h}$ is bounded at tree-level by $m_{Z}$, requiring $m_{h} \simeq 125 \mathrm{GeV}$ implies a lower bound $\hat{m}_{\tilde{t}}^{2} \gtrsim 550 \mathrm{GeV}$ for $X_{t}^{\max }$. While this is the main source of fine-tuning in the MSSM, it can be easily evaded in e.g. non-minimal SUSY models, where the correct Higgs mass can be obtained via non-decoupling $D$-terms [155], or in the next-to-minimal supersymmetric SM with special parameter choices [156].

In figure 12 the SI cross section is displayed as a function of the lightest stop mass $m_{\tilde{t}_{1}}$ for $\tan \beta=10$ and several values of $\mu$. We find that a positive value of $\mu$ is excluded by LUX, while negative values become progressively harder to constrain as the mass difference between the bino and Higgsinos is increased. The blind spot is clearly seen for $\mu=-4 M_{1}$ and occurs at a light stop mass $m_{\tilde{t}_{1}} \simeq 160 \mathrm{GeV}$. 


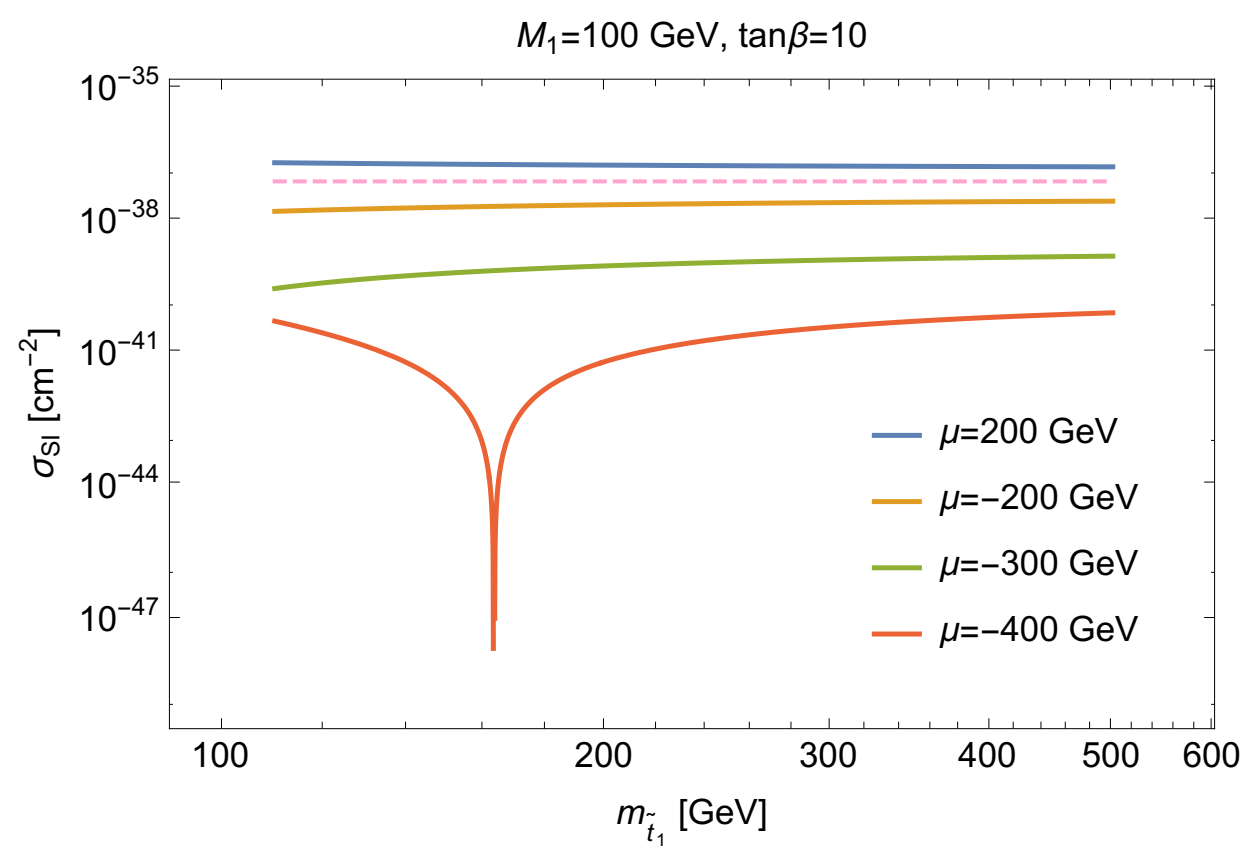

Figure 12. SI $\chi$-xenon cross sections as a function of the lightest stop mass $m_{\tilde{t}_{1}}$. The solid lines correspond to different values of the Higgsino mass $\mu$, while the pink dashed line is the existing limit from LUX. The blind spot at $m_{\tilde{t}_{1}} \simeq 160 \mathrm{GeV}$ is shown in red.

In figure 13 we show the interplay between collider and DM direct-detection limits in the $\left(m_{\tilde{t}_{1}}, M_{1}\right)$ plane for two values of $\tan \beta$ and $\mu$. We find that in the absence of blind spots $\left(\mu=-2 M_{1}\right)$, LUX excludes the $M_{1} \lesssim 50 \mathrm{GeV}$ region across a large range of stop masses. These limits will be significantly improved if XENON1T and LZ do not detect a DM signal, with whole regions below $M_{1} \approx 300 \mathrm{GeV}$ and $500 \mathrm{GeV}$ excluded by the respective experiments. In these cases, the direct-detection limits surpass those derived from the ATLAS searches for stops and sbottoms.

In the blind spot region $\left(\mu=-4 M_{1}\right)$, the DM limits are considerably weakened, with LZ excluding most of the $M_{1} \lesssim 50 \mathrm{MeV}$ and $150 \mathrm{MeV}$ regions for $\tan \beta=10$ and 25 respectively. As noted in section 3.2, isospin violation can be enhanced in the proximity of blind spots which arise from destructive interference in the amplitude. Since this is a generic feature of SI scattering, it follows that isospin violation can also be large near the blind spots shown in figure 13. Although these regions are excluded by collider limits, we have not ruled out the possibility that blind spots for this model occur in viable regions of parameter space.

\subsection{Generic Higgs and light squark exchange}

In this section, we consider the effect of adding $H$ to the particle content, so that the active degrees of freedom are $h, H$ and $\tilde{t}_{1,2}, \tilde{b}_{L}$ [model (D) in figure 3]. This is the most "natural" model studied in this article since a large value of $m_{H}$ would also require fine tuning [121].

To derive an analytic formula for the blind spot, we follow the same steps used to obtain (3.10). For moderate to large values of $m_{A}>m_{h}$ and $\tan \beta$, we find that a blind 

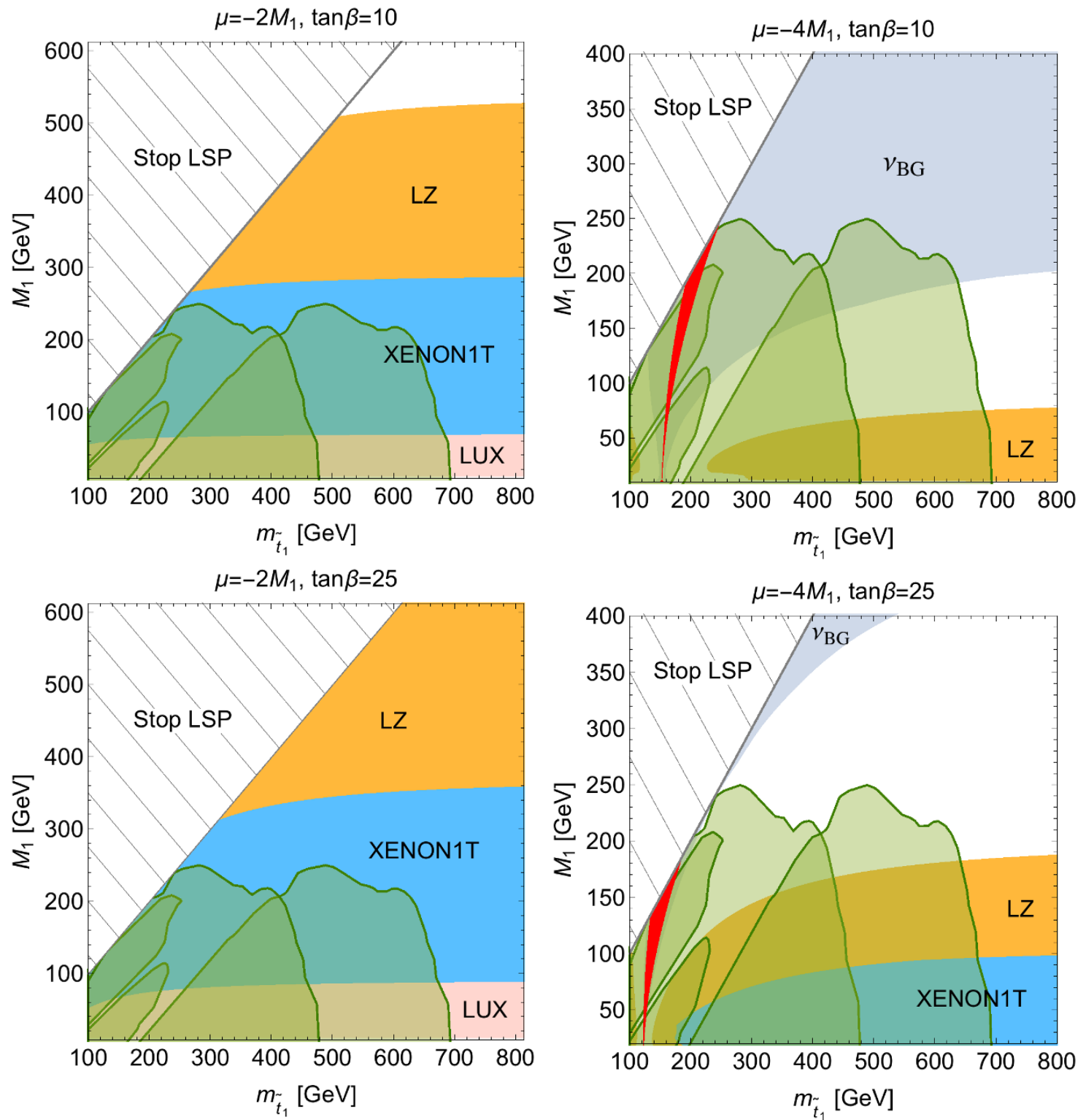

Figure 13. Current and projected limits due to light Higgs $h$ and squark $\tilde{t}_{1,2}, \tilde{b}_{L}$ exchange in $\chi$ xenon scattering with $\tan \beta=10$ (top row) and $\tan \beta=25$ (bottom row) and two benchmarks for negative $\mu$. The figures show the DM constraints from LUX [21] and XENON1T [22] (color coded as in figure 4), while limits from direct searches for stops and sbottoms at ATLAS [157] are shown in green. The blind spot is shown in red and lies within the neutrino background $\left(\nu_{\mathrm{BG}}\right)$ shown in gray. The hatched region corresponds to the case where $\tilde{t}_{1}$ becomes the LSP. 
spot occurs whenever

$$
\frac{30}{m_{h}^{2}}\left(M_{1}+\mu s_{2 \beta}\right)+\mu \tan \beta\left(\frac{15}{m_{H}^{2}}+\frac{1}{m_{\tilde{b}_{L}}^{2}-M_{1}^{2}}\right) \simeq 0
$$

is satisfied. In this case, the inclusion of $H$ has the effect of shifting the location of the blind spot found for $h$ and squark exchange (3.11). In particular, negative values of $\mu$ are still required.

To examine the limits on this model, we must also consider flavor observables since $H$ and light stops contribute to $B \rightarrow X_{s} \gamma$ and $B_{s} \rightarrow \mu^{+} \mu^{-}$. To evaluate the flavor constraints we use SUSY_FLAVOR-2.51 [158-160] and implement the NNLO SM calculation by constraining the ratio

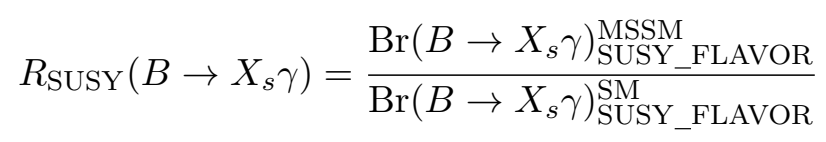

to lie within the allowed range for

$$
R_{\mathrm{EXP}}\left(B \rightarrow X_{s} \gamma\right)=\frac{\operatorname{Br}\left(B \rightarrow X_{s} \gamma\right)_{\mathrm{EXP}}}{\operatorname{Br}\left(B \rightarrow X_{s} \gamma\right)_{\mathrm{NNLO}}^{\mathrm{SM}}}
$$

Here we use the recent calculation of [154]

$$
\operatorname{Br}\left(B \rightarrow X_{s} \gamma\right)_{\mathrm{SM}}=(3.36 \pm 0.23) \times 10^{-4}
$$

to incorporate the NNLO SM prediction. For the experimental value we use the PDG average [161] within $2 \sigma$ uncertainties,

$$
\operatorname{Br}\left(B \rightarrow X_{s} \gamma\right)_{\mathrm{EXP}}=(3.41 \pm 0.21 \pm 0.07) \times 10^{-4} .
$$

We add the theoretical error linearly with the experimental one, so that $R_{\mathrm{SUSY}}$ is required to lie within the interval

$$
R_{\mathrm{EXP}}^{\mathrm{MIN}}\left(B \rightarrow X_{s} \gamma\right) \leq R_{\mathrm{SUSY}}\left(B \rightarrow X_{s} \gamma\right) \leq R_{\mathrm{EXP}}^{\mathrm{MAX}}\left(B \rightarrow X_{s} \gamma\right)
$$

For $B_{s} \rightarrow \mu^{+} \mu^{-}$we adopt the same procedure to impose limits on the relevant SUSY parameter space. Here the SM prediction [162] is

$$
\operatorname{Br}\left(B_{s} \rightarrow \mu^{+} \mu^{-}\right)_{\mathrm{SM}}=(3.65 \pm 0.23) \times 10^{-9},
$$

and has to be compared against

$$
\operatorname{Br}\left(B_{s} \rightarrow \mu^{+} \mu^{-}\right) \operatorname{EXP}=(3.1 \pm 0.7) \times 10^{-9} .
$$

In figure 14 we display limits in the $\left(m_{\tilde{t}_{1}}, M_{1}\right)$ plane for $\tan \beta=10$ and several values of $m_{A}$. Since this choice of $\tan \beta$ corresponds to a horizontal slice through the $h, H$ parameter space (figure 8), the effect of increasing $m_{A}$ is to probe the effect of the $h, H$ blind spot (3.9) from below. For $m_{A}=300 \mathrm{GeV}$ and away from the blind spot, we find that LUX excludes 

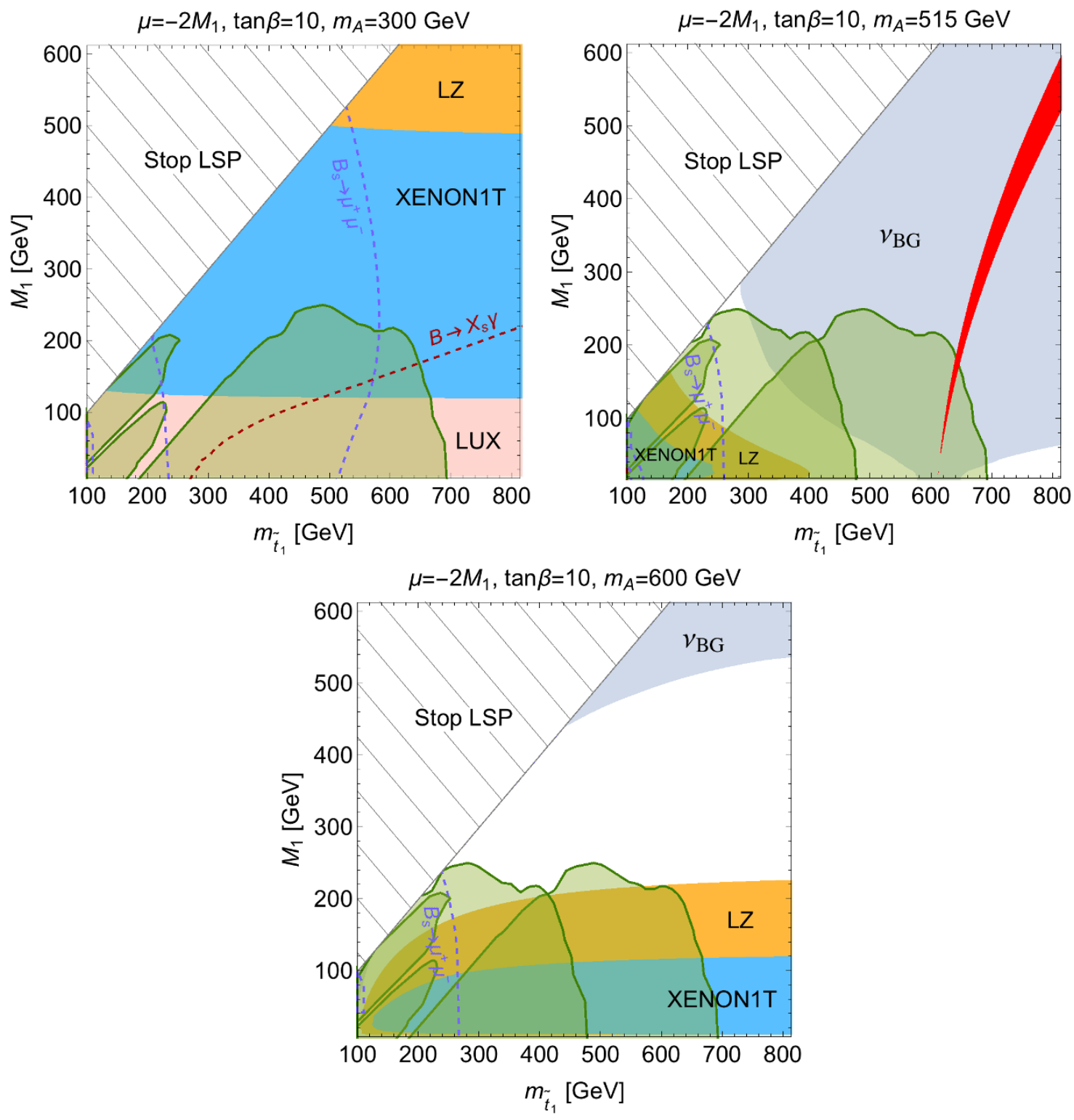

Figure 14. Current and projected limits in the $\left(m_{\tilde{t}_{1}}, M_{1}\right)$ plane from $h, H$ and $\tilde{t}_{1,2}, \tilde{b}_{L}$ exchange in $\chi$-xenon scattering. The three plots represent different values of $m_{A}$, for fixed $\tan \beta=10$. For $A_{t}^{\max }<0$, the regions between the purple dashed lines are excluded by $B_{s} \rightarrow \mu^{+} \mu^{-}$, while regions to the left of the dark red dashed lines are excluded by $B \rightarrow X_{s} \gamma$. Excluded regions from direct detection are color-coded as in figure 13. 

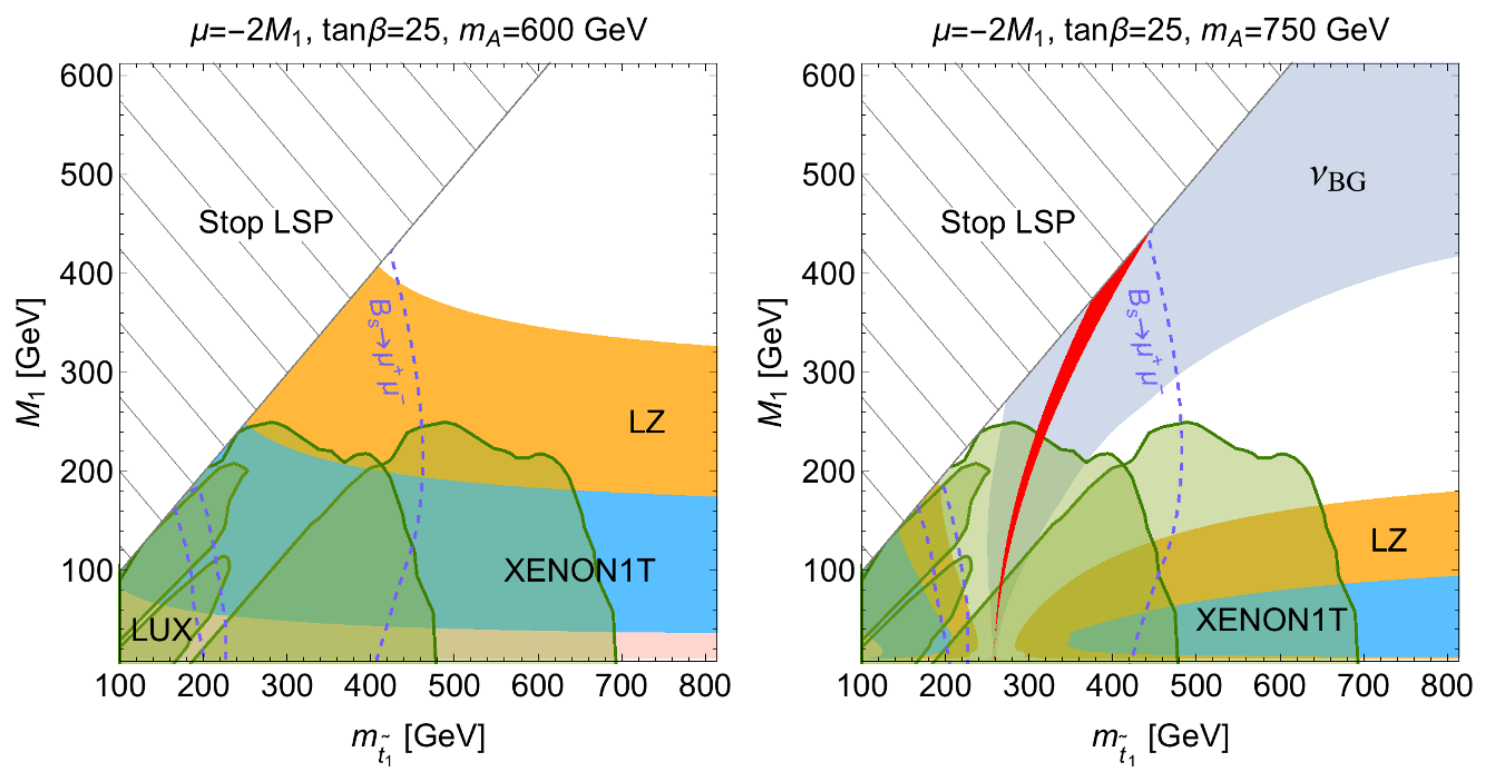

Figure 15. Current and projected limits in the $\left(m_{\tilde{t}_{1}}, M_{1}\right)$ plane from $h, H$ and $\tilde{t}_{1,2}, \tilde{b}_{L}$ exchange in $\chi$-xenon scattering. In the figures, the value of $m_{A}$ is increased for fixed $\tan \beta$. Excluded regions are color-coded as in figure 14, with $B \rightarrow X_{s} \gamma$ ruling out both values of $m_{A}$ entirely.

the band below $M_{1} \approx 100 \mathrm{GeV}$. In this case, the limits from $B \rightarrow X_{s} \gamma$ and $B_{s} \rightarrow \mu^{+} \mu^{-}$ provide a complementary and stringent constraint: compatibility with both observables and LUX only leaves a small region of parameter space viable. XENON1T and LZ will carve out most of the remaining parameter space, providing a very strong constraint on the light $m_{A}$ scenario. However, for heavier values of $m_{A}$, the constraints from flavor and direct detection weaken considerably and here the collider bounds become the dominant constraint. This is particularly evident in the second plot of figure 14, where the blind spot suppresses the SI cross section and the moderate value of $m_{A}$ reduces the tension with $B \rightarrow X_{s} \gamma$ entirely.

We consider the effect of increasing $\tan \beta$ in figure 15 . For $m_{A}=600 \mathrm{GeV}$, there is no blind spot in the physical region, with most of the area below $m_{\tilde{t}_{1}} \approx 450 \mathrm{MeV}$ excluded, while for $m_{A}=750 \mathrm{GeV}$ a blind spot does occur. This latter region is allowed by current collider limits, but excluded by $B \rightarrow X_{s} \gamma$. However, one should keep in mind that the flavor bounds included in figure 14 (and figure 15) are the least rigorous ones as they can be evaded if some of the underlying assumptions are relaxed: in the presence of non-minimal sources of flavor violation the bounds can become weaker. In fact, a mass splitting among the left-handed squarks deviates from naive minimal flavor violation since there are either off-diagonal elements in the up or in the down sector of the squark mass matrix (or in both simultaneously). Furthermore, relaxing our assumption that the left-handed bilinear terms are diagonal in the down basis (2.18) would lead to additional effects in flavor observables. 


\section{Conclusions}

In this paper we examined four simplified models in the framework of the MSSM where all but a handful of superpartners are decoupled from the spectrum. We started from the minimal model necessary to provide a viable DM candidate and sequentially added particles to render the spectrum more natural. The key result, analytic expressions for the Wilson coefficients relevant for the Higgs- and squark-exchange contribution to spin-independent WIMP-nucleon scattering, is summarized in (2.24)-(2.27).

As the main application of our scheme, we studied the amount of isospin violation generated by single-nucleon contributions to the spin-independent WIMP-nucleon cross section. In general, isospin violation is a rather small effect: for pure $h$ exchange, the amount of isospin violation is $\approx 5 \%$, i.e. in line with common expectations and results in two-Higgs-doublet models [163]. Beyond single $h$ exchange, however, the effect can be enhanced in the proximity of blind spots. In such cases, the blind spots occur (at a given order in perturbation theory) due to destructive interference among different contributions to the SI amplitudes. As a consequence, small variations of the amplitude become increasingly important. Although the SI cross sections are strongly suppressed in the vicinity of blind spots, as direct-detection experiments become increasingly more sensitive, MSSM models are pushed towards these corners of parameter space. For instance, we find that for $h$ and $H$ exchange, the projected limits from LZ [23] allow isospin violation to be as large as $40 \%$, which increases rapidly as one approaches the irreducible neutrino background. In this way, precise predictions for isospin violation are essential to relate future direct-detection data to MSSM predictions.

In our simplified models, the source of isospin violation originates purely from the $\mathrm{SM}$; it is the blind spots that make these small differences prominent. This situation is unlike e.g. in $Z^{\prime}$ models which introduce new sources of isospin violation beyond the SM. Therefore, an accurate evaluation of isospin violation requires a careful assessment of the nuclear input quantities and the associated hadronic uncertainties. We demonstrated this point by comparing different methods to determine the proton and neutron scalar matrix elements that are currently used in the literature. While the traditional approach based on $\chi \mathrm{PT}_{3}$ relations suffers from large and, in part, uncontrolled uncertainties, the hadronic input can be accurately evaluated by using the two-flavor formalism developed in [66]. In particular, we showed that in the three-flavor framework, depending on which input is used for the strangeness-related quantities, incorrect conclusions concerning both central values and uncertainties can occur.

We also extended our models to include light stops and sbottoms in the spectrum. Again, for certain corners of the parameter space, cancellations occur that suppress the amplitude and led us to the identification of new blind spots. We identified these blind spots analytically in (3.11) and (3.15). Furthermore, the interplay between DM, collider, and flavor limits was studied, finding that the inclusion of the latter tends to exclude configurations with blind spots which are allowed by collider bounds. Only for $\tan \beta=10$ and near the blind spot generated by $h$ and $H$ exchange, did we find a blind spot consistent with all constraints (figure 14). 


\section{Acknowledgments}

We thank Ross Young and Joel Giedt for useful correspondence regarding [99], Ahmed Ismail and Thomas Rizzo for providing us with details of their pMSSM benchmark models [164], Geneviève Bélanger and Alexander Pukhov for correspondence concerning MICROMEGAs [67], and Xavier Garcia i Tormo and DingYu Shao for useful discussions. Financial support by BMBF ARCHES, the Helmholtz Alliance HA216/EMMI, and the Swiss National Science Foundation (SNF) is gratefully acknowledged. A.C. is supported by a Marie Curie Intra-European Fellowship of the European Community's 7th Framework Programme under contract number (PIEF-GA-2012-326948).

\section{A Perturbative diagonalization of the neutralino mass matrix}

In this appendix, we diagonalize the neutralino mass matrix $M^{\chi}(2.16)$ via a perturbative expansion in $v / M_{\mathrm{SUSY}}$. Following [108-110], we first consider the diagonal matrix $Z_{a}^{\chi \dagger} M^{\chi \dagger} M^{\chi} Z_{a}^{\chi}$, where to leading order in $v / M_{\mathrm{SUSY}}$ we have

$$
Z_{a}^{\chi}=\left(\begin{array}{cccc}
1 & 0 & \frac{g_{1} v}{\sqrt{2}} \frac{\left(M_{1}^{*} c_{\beta}+\mu s_{\beta}\right)}{\left|M_{1}\right|^{2}-|\mu|^{2}} & -\frac{g_{1} v}{\sqrt{2}} \frac{\left(M_{1}^{*} s_{\beta}+\mu c_{\beta}\right)}{\left|M M_{1}\right|^{2}-|\mu|^{2}} \\
0 & 1 & -\frac{g_{2} v}{\sqrt{2}} \frac{\left(M_{1}^{*} \beta_{\beta}+\mu \mu_{\beta}\right)}{\left|M_{1}\right|^{2}-|\mu|^{2}} & \frac{g_{2} v}{\sqrt{2}} \frac{\left(M_{2}^{*} s_{\beta}+\mu c_{\beta}\right)}{\left|M_{1}\right|^{2}-|\mu|^{2}} \\
-\frac{g_{1} v}{\sqrt{2}} \frac{\left(M_{1} c_{\beta}+\mu^{*} s_{\beta}\right)}{\left|M_{1}\right|^{2}-|\mu|^{2}} & \frac{g_{2} v}{\sqrt{2}} \frac{\left(M_{1} c_{\beta}+\mu^{*} s_{\beta}\right)}{\left|M_{1}\right|^{2}-|\mu|^{2}} & 1 & 0 \\
\frac{g_{2} v}{\sqrt{2}} \frac{\left(M_{1} s_{\beta}+\mu^{*} c_{\beta}\right)}{\left|M_{1}\right|^{2}-|\mu|^{2}} & -\frac{g_{2} v}{\sqrt{2}} \frac{\left(M_{2} s_{\beta}+\mu^{*} c_{\beta}\right)}{\left|M_{1}\right|^{2}-|\mu|^{2}} & 0 & 1
\end{array}\right) .
$$

Although $Z_{a}^{\chi}$ diagonalizes the square $M^{\chi \dagger} M^{\chi}$, we need to perform two additional rotations in order to make $Z^{\chi^{T}} M^{\chi} Z^{\chi}$ real and diagonal:

$$
Z_{b}^{\chi}=\left(\begin{array}{cccc}
1 & 0 & 0 & 0 \\
0 & 1 & 0 & 0 \\
0 & 0 & \frac{1}{\sqrt{2}} & -\frac{1}{\sqrt{2}} \\
0 & 0 & \frac{1}{\sqrt{2}} & \frac{1}{\sqrt{2}}
\end{array}\right) \quad \text { and } \quad Z_{c}^{\chi}=\left(\begin{array}{cccc}
e^{-i \phi_{M_{1}} / 2} & 0 & 0 & 0 \\
0 & e^{-i \phi_{M_{2}} / 2} & 0 & 0 \\
0 & 0 & e^{-i \phi_{\mu} / 2} & 0 \\
0 & 0 & 0 & e^{-i \phi_{\mu} / 2}
\end{array}\right)
$$

where $\phi_{M_{i}, \mu}$ is the phase of $M_{1,2}$ and $\mu$ respectively. The resulting mixing matrix is given by

$$
Z^{\chi}=Z_{a}^{\chi} Z_{b}^{\chi} Z_{c}^{\chi}
$$

from which we deduce the relevant components for the lightest neutralino

$$
\begin{aligned}
& Z_{11}^{\chi}=e^{-\frac{i}{2} \phi_{M_{1}}}+O\left(v^{2} / M_{\mathrm{SUSY}}^{2}\right) \\
& Z_{21}^{\chi}=O\left(v^{2} / M_{\mathrm{SUSY}}^{2}\right), \\
& Z_{31}^{\chi}=-\frac{e^{-\frac{i}{2} \phi_{M_{1}}}}{\sqrt{2}} \frac{g_{1} v}{\left|M_{1}\right|^{2}-|\mu|^{2}}\left(M_{1} c_{\beta}+\mu^{*} s_{\beta}\right)+O\left(v^{2} / M_{\mathrm{SUSY}}^{2}\right), \\
& Z_{41}^{\chi}=\frac{e^{-\frac{i}{2} \phi_{M_{1}}}}{\sqrt{2}} \frac{g_{1} v}{\left|M_{1}\right|^{2}-|\mu|^{2}}\left(M_{1} s_{\beta}+\mu^{*} c_{\beta}\right)+O\left(v^{2} / M_{\mathrm{SUSY}}^{2}\right) .
\end{aligned}
$$




\section{B Analytic expressions for $\chi$-nucleon scattering}

This appendix concerns the derivation of the analytic expressions (2.24)-(2.27) for the Wilson coefficients $C_{q_{i}}$ appearing in the scalar $\chi$-nucleon couplings (2.2). The derivation involves an analysis of the spin-independent (SI) amplitude for $\chi q_{i} \rightarrow \chi q_{i}$ scattering due to tree-level Higgs and squark exchange (figure 2).

Squark exchange. Consider first the contribution due to squark exchange, where the zero-momentum propagator for the $s$ - and $u$-channels is denoted by

$$
D_{q_{i} \tilde{q}_{s}}^{ \pm}=\frac{1}{\left(m_{\chi} \pm m_{q_{i}}\right)^{2}-m_{\tilde{q}_{s}}^{2}+i \epsilon}
$$

and we define

$$
i\left(\Gamma_{L}^{q_{i} \tilde{q}_{s} *} P_{R}+\Gamma_{R}^{q_{i} \tilde{q}_{s} *} P_{L}\right)
$$

as the Feynman rule for the $\chi \bar{q}_{i} \tilde{q}_{s}$ coupling. Then for spinors $u_{i}$ and $v_{i}$ carrying momentum $p_{i}$, the $s$-channel amplitude is

$$
\begin{aligned}
\mathcal{A}_{s}^{q_{i}}= & \sum_{s=1}^{6} \bar{v}_{1}\left[i\left(\Gamma_{L}^{q_{i} \tilde{q}_{s} *} P_{R}+\Gamma_{R}^{q_{i} \tilde{q}_{s} *} P_{L}\right)\right] u_{2}\left[i D_{q_{i}}^{+} \tilde{q}_{s}\right] \bar{u}_{4}\left[i\left(\Gamma_{L}^{q_{i} \tilde{q}_{s}} P_{L}+\Gamma_{R}^{q_{i} \tilde{q}_{s}} P_{R}\right)\right] v_{3} \\
= & -\frac{i}{2} \sum_{s=1}^{6} D_{q_{i}}^{+} \tilde{q}_{s}\left\{\Gamma_{L}^{q_{i} \tilde{q}_{s} *} \Gamma_{L}^{q_{i} \tilde{q}_{s}}\left(\bar{v}_{1} \gamma^{\mu} P_{L} v_{3}\right)\left(\bar{u}_{4} \gamma_{\mu} P_{R} u_{2}\right)+\Gamma_{R}^{q_{i} \tilde{q}_{s} *} \Gamma_{R}^{q_{i} \tilde{q}_{s}}\left(\bar{v}_{1} \gamma^{\mu} P_{R} v_{3}\right)\left(\bar{u}_{4} \gamma_{\mu} P_{L} u_{2}\right)\right. \\
& +\Gamma_{L}^{q_{i} \tilde{q}_{s} *} \Gamma_{R}^{q_{i} \tilde{q}_{s}}\left[\left(\bar{v}_{1} P_{R} v_{3}\right)\left(\bar{u}_{4} P_{R} u_{2}\right)+\frac{1}{4}\left(\bar{v}_{1} \sigma^{\mu \nu} v_{3}\right)\left(\bar{u}_{4} \sigma_{\mu \nu} P_{R} u_{2}\right)\right] \\
& \left.+\Gamma_{R}^{q_{i} \tilde{q}_{s} *} \Gamma_{L}^{q_{i} \tilde{q}_{s}}\left[\left(\bar{v}_{1} P_{L} v_{3}\right)\left(\bar{u}_{4} P_{L} u_{2}\right)+\frac{1}{4}\left(\bar{v}_{1} \sigma^{\mu \nu} v_{3}\right)\left(\bar{u}_{4} \sigma_{\mu \nu} P_{L} u_{2}\right)\right]\right\}
\end{aligned}
$$

where the Fierz identities

$$
\begin{aligned}
\left(P_{L, R}\right)_{i j}\left(P_{L, R}\right)_{k l} & =\frac{1}{2}\left(P_{L, R}\right)_{i l}\left(P_{L, R}\right)_{k j}+\frac{1}{8}\left(\sigma^{\mu \nu}\right)_{i l}\left(\sigma_{\mu \nu} P_{L, R}\right)_{k j}, \\
\left(P_{L, R}\right)_{i j}\left(P_{R, L}\right)_{k l} & =\frac{1}{2}\left(\gamma^{\mu} P_{R, L}\right)_{i l}\left(\gamma_{\mu} P_{L, R}\right)_{k j},
\end{aligned}
$$

have been used to obtain the final equality. Similarly, in the $u$-channel we find

$$
\begin{aligned}
\mathcal{A}_{u}^{q_{i}}= & -\sum_{s=1}^{6} \bar{v}_{3}\left[i\left(\Gamma_{L}^{q_{i} \tilde{q}_{s}} P_{L}+\Gamma_{R}^{q_{i} \tilde{q}_{s}} P_{R}\right)\right] u_{2}\left[i D_{q_{i}}^{-} \tilde{q}_{s}\right] \bar{u}_{4}\left[i\left(\Gamma_{L}^{q_{i} \tilde{q}_{s} *} P_{R}+\Gamma_{R}^{q_{i} \tilde{q}_{s} *} P_{L}\right)\right] v_{1} \\
= & \frac{i}{2} \sum_{s=1}^{6} D_{q_{i}}^{-} \tilde{q}_{s}\left\{\Gamma_{L}^{q_{i} \tilde{q}_{s} *} \Gamma_{L}^{q_{i} \tilde{q}_{s}}\left(\bar{v}_{3} \gamma^{\mu} P_{R} v_{1}\right)\left(\bar{u}_{4} \gamma_{\mu} P_{L} u_{2}\right)+\Gamma_{R}^{q_{i} \tilde{q}_{s} *} \Gamma_{R}^{q_{i} \tilde{q}_{s}}\left(\bar{v}_{3} \gamma^{\mu} P_{L} v_{1}\right)\left(\bar{u}_{4} \gamma_{\mu} P_{R} u_{2}\right)\right. \\
& +\Gamma_{L}^{q_{i} \tilde{q}_{s}} \Gamma_{R}^{q_{i} \tilde{q}_{s} *}\left[\left(\bar{v}_{3} P_{L} v_{1}\right)\left(\bar{u}_{4} P_{L} u_{2}\right)+\frac{1}{4}\left(\bar{v}_{3} \sigma^{\mu \nu} v_{1}\right)\left(\bar{u}_{4} \sigma_{\mu \nu} P_{L} u_{2}\right)\right] \\
& \left.+\Gamma_{R}^{q_{i} \tilde{q}_{s}} \Gamma_{L}^{q_{i} \tilde{q}_{s} *}\left[\left(\bar{v}_{3} P_{R} v_{1}\right)\left(\bar{u}_{4} P_{R} u_{2}\right)+\frac{1}{4}\left(\bar{v}_{3} \sigma^{\mu \nu} v_{1}\right)\left(\bar{u}_{4} \sigma_{\mu \nu} P_{R} u_{2}\right)\right]\right\}
\end{aligned}
$$


so neglecting the spin-dependent terms involving $\gamma_{\mu}$ and $\gamma_{\mu} \gamma_{5}$ gives,

$$
\left.\mathcal{A}_{s+u}^{q_{i}}\right|_{\mathrm{SI}}=\frac{i}{4} \sum_{s=1}^{6}\left[D_{q_{i}}^{+} \tilde{q}_{s}+D_{q_{i} \tilde{q}_{s}}^{-}\right] \operatorname{Re}\left\{\Gamma_{L}^{q_{i} \tilde{q}_{s}} \Gamma_{R}^{q_{i} \tilde{q}_{s} *}\right\}\left(\bar{v}_{3} v_{1}\right)\left(\bar{u}_{4} u_{2}\right)+\gamma_{5} \text { terms } .
$$

At zero-momentum transfer, the terms involving $\gamma_{5}$ are suppressed and so we can read off the Wilson coefficient for the operator $\bar{\chi} \chi \bar{q}_{i} q_{i}$ :

$$
\begin{aligned}
\bar{m}_{q_{i}} C_{q_{i}}^{\tilde{q}} & =\frac{1}{8} \sum_{s=1}^{6}\left[D_{q_{i} \tilde{q}_{s}}^{+}+D_{q_{i} \tilde{q}_{s}}^{-}\right] \operatorname{Re}\left\{\Gamma_{L}^{q_{i} \tilde{q}_{s}} \Gamma_{R}^{q_{i} \tilde{q}_{s} *}\right\} \\
& =\frac{1}{8} \sum_{s=1}^{6}\left[\frac{1}{\left(m_{\chi}+m_{q_{i}}\right)^{2}-m_{\tilde{q}_{s}}^{2}+i \epsilon}+\frac{1}{\left(m_{\chi}-m_{q_{i}}\right)^{2}-m_{\tilde{q}_{s}}^{2}+i \epsilon}\right] \operatorname{Re}\left\{\Gamma_{L}^{q_{i} \tilde{q}_{s}} \Gamma_{R}^{q_{i} \tilde{q}_{s} *}\right\},
\end{aligned}
$$

where there is no sum over $q_{i}$ and $\bar{m}_{q_{i}}$ is the running quark mass. In the literature, the quark mass in $D_{q_{i}}^{ \pm} \tilde{q}_{s}$ is often neglected, in which case the $s$ - and $u$-channel amplitudes coincide with each other. Note that by substituting the couplings [103]

$$
\begin{aligned}
\Gamma_{L}^{d_{i} \tilde{d}_{s}} & =\sqrt{2} g_{2}\left(\frac{1}{2} Z_{21}^{\chi}-\frac{1}{6} \tan \theta_{W} Z_{11}^{\chi}\right) Z_{i s}^{\tilde{d} *}-Y_{d_{i}}^{*} Z_{31}^{\chi} Z_{i+3, s}^{\tilde{d} *}, \\
\Gamma_{R}^{d_{i} \tilde{d}_{s}} & =-\frac{\sqrt{2}}{3} g_{2} \tan \theta_{W} Z_{11}^{\chi *} Z_{i+3, s}^{\tilde{d} *}-Y_{d_{i}} Z_{31}^{\chi *} Z_{i s}^{\tilde{d} *}, \\
\Gamma_{L}^{u_{i} \tilde{u}_{s}} & =-\sqrt{2} g_{2}\left(\frac{1}{2} Z_{21}^{\chi}+\frac{1}{6} \tan \theta_{W} Z_{11}^{\chi}\right) Z_{i s}^{\tilde{u} *}-Y_{u_{i}}^{*} Z_{41}^{\chi} Z_{i+3, s}^{\tilde{u} *}, \\
\Gamma_{R}^{u_{i} \tilde{u}_{s}} & =\frac{2 \sqrt{2}}{3} g_{2} \tan \theta_{W} Z_{11}^{\chi *} Z_{i+3, s}^{\tilde{u} *}-Y_{u_{i}} Z_{41}^{\chi *} Z_{i s}^{\tilde{u} *}
\end{aligned}
$$

into (B.7), one recovers the expressions given in [105].

To simplify (B.7), we expand all mixing matrices in powers of $v / M_{\mathrm{SUSY}}$. At leading order, the elements $Z_{I J}^{\chi}$ are given by (2.23), while products of squark mixing matrices simplify as follows [165]:

$$
\begin{aligned}
\sum_{s=1}^{6} Z_{i s}^{\tilde{u}} Z_{i+3, s}^{\tilde{u} *} D_{q_{i} \tilde{q}_{s}}^{ \pm} & =\Delta_{u_{i}} \frac{L_{u_{i}}^{ \pm}-R_{u_{i}}^{ \pm}}{m_{\tilde{u}_{i}^{L}}^{2}-m_{\tilde{u}_{i}^{R}}^{2}} \\
\sum_{s=1}^{6} Z_{i s}^{\tilde{d}} Z_{i+3, s}^{\tilde{d} *} D_{q_{i} \tilde{q}_{s}}^{ \pm} & =\Delta_{d_{i}} \frac{L_{d_{i}}^{ \pm}-R_{d_{i}}^{ \pm}}{m_{\tilde{d}_{i}^{L}}^{2}-m_{\tilde{d}_{i}^{R}}^{2}} \\
\sum_{s=1}^{6} Z_{i+3, s}^{\tilde{d}} Z_{i+3, s}^{\tilde{d} *} D_{q_{i} \tilde{q}_{s}}^{ \pm} & =R_{d_{i}}^{ \pm} \\
\sum_{s=1}^{6} Z_{i s}^{\tilde{d}} Z_{i s}^{\tilde{d} *} D_{q_{i}}^{ \pm} \tilde{q}_{s} & =L_{d_{i}}^{ \pm} \\
\sum_{s=1}^{6} Z_{i+3, s}^{\tilde{u}} Z_{i+3, s}^{\tilde{u} *} D_{q_{i} \tilde{q}_{s}}^{ \pm} & =R_{u_{i}}^{ \pm} \\
\sum_{s=1}^{6} Z_{i s}^{\tilde{u}} Z_{i s}^{\tilde{u} *} D_{q_{i} \tilde{q}_{s}}^{ \pm} & =L_{u_{i}}^{ \pm},
\end{aligned}
$$


where $m_{\tilde{q}_{i}^{L}}^{2}$ and $m_{\tilde{q}_{i}^{R}}^{2}$ correspond to the upper and lower diagonal elements of the squark $(\text { mass })^{2}$ matrices $(2.18)$,

$$
\Delta_{u_{i}}=-\bar{m}_{u_{i}}\left(A_{u}^{i i}+\mu \cot \beta\right) \quad \text { and } \quad \Delta_{d_{i}}=-\bar{m}_{d_{i}}\left(A_{d}^{i i}+\mu \tan \beta\right),
$$

are the off-diagonal elements, and the squark propagators in the chiral basis are

$$
S_{q_{i}}^{ \pm}=\frac{1}{\left(m_{\chi} \pm m_{q_{i}}\right)^{2}-m_{\tilde{q}_{i}^{S}}^{2}+i \epsilon}, \quad \text { for } S=L \text { or } R
$$

Neglecting terms of $O\left(v / M_{\mathrm{SUSY}}^{2}\right)$, the final result is

$$
\begin{aligned}
& \bar{m}_{u_{i}} C_{u_{i}}^{\tilde{q}}=\frac{1}{8} \operatorname{Re}\left\{-\frac{4}{3} g_{2}^{2} \tan \theta_{W}\left(\frac{1}{2} Z_{11}^{\chi *} Z_{21}^{\chi *}+\frac{1}{6} \tan \theta_{W} Z_{11}^{\chi *} Z_{11}^{\chi *}\right) \Delta_{u_{i}} L_{u_{i}}^{+} R_{u_{i}}^{+}\right.
\end{aligned}
$$

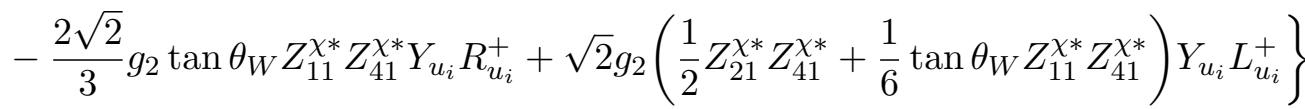

$$
\begin{aligned}
& +\left(L^{+}, R^{+}\right) \leftrightarrow\left(L^{-}, R^{-}\right) \\
& =\bar{m}_{u_{i}} \frac{g_{1}^{2}}{8} \operatorname{Re}\left\{e^{i \phi_{M_{1}}}\left[\frac{2}{9} X_{u_{i}} L_{u_{i}}^{+} R_{u_{i}}^{+}+\frac{1}{6} \frac{\left(M_{1}^{*}+\mu \cot \beta\right)}{\left|M_{1}\right|^{2}-|\mu|^{2}}\left(L_{u_{i}}^{+}-4 R_{u_{i}}^{+}\right)\right]\right\} \\
& +\left(L^{+}, R^{+}\right) \leftrightarrow\left(L^{-}, R^{-}\right), \\
& \bar{m}_{d_{i}} C_{d_{i}}^{\tilde{q}}=\frac{1}{8} \operatorname{Re}\left\{-\frac{2}{3} g_{2}^{2} \tan \theta_{W}\left(\frac{1}{2} Z_{21}^{\chi *} Z_{11}^{\chi *}-\frac{1}{6} \tan \theta_{W} Z_{11}^{\chi *} Z_{11}^{\chi *}\right) \Delta_{d_{i}} L_{d_{i}}^{+} R_{d_{i}}^{+}\right. \\
& \left.-\sqrt{2} g_{2}\left(\frac{1}{2} Z_{21}^{\chi *} Z_{31}^{\chi *}-\frac{1}{6} \tan \theta_{W} Z_{11}^{\chi *} Z_{31}^{\chi *}\right) Y_{d_{i}} L_{d_{i}}^{+}+\frac{\sqrt{2}}{3} g_{2} \tan \theta_{W} Z_{11}^{\chi *} Z_{31}^{\chi_{1}} Y_{d_{i}} R_{d_{i}}^{+}\right\} \\
& +\left(L^{+}, R^{+}\right) \leftrightarrow\left(L^{-}, R^{-}\right) \\
& =-\bar{m}_{d_{i}} \frac{g_{1}^{2}}{8} \operatorname{Re}\left\{e^{i \phi_{M_{1}}}\left[\frac{1}{9} X_{d_{i}} L_{d_{i}}^{+} R_{d_{i}}^{+}+\frac{1}{6} \frac{\left(M_{1}^{*}+\mu \tan \beta\right)}{\left|M_{1}\right|^{2}-|\mu|^{2}}\left(L_{d_{i}}^{+}+2 R_{d_{i}}^{+}\right)\right]\right\} \\
& +\left(L^{+}, R^{+}\right) \leftrightarrow\left(L^{-}, R^{-}\right)
\end{aligned}
$$

where the squark mixing $X_{q_{i}}$ is defined in (2.28).

Higgs exchange. Now consider the Higgs contribution, for which the $t$-channel amplitude reads

$$
\begin{aligned}
\mathcal{A}_{t}^{h, H} & =\sum_{k=1,2} \bar{u}_{3}\left[i\left(\Gamma_{\chi \chi}^{H_{k}} P_{R}+\Gamma_{\chi \chi}^{H_{k} *} P_{L}\right)\right] u_{1}\left[\frac{i}{-m_{H_{k}}^{2}}\right] \bar{u}_{4}\left[i\left(\Gamma_{q_{i} q_{i}}^{H_{k}} P_{R}+\Gamma_{q_{i} q_{i}}^{H_{k} *} P_{L}\right)\right] u_{2} \\
& =i \sum_{k=1,2} \frac{1}{m_{H_{k}}^{2}} \operatorname{Re}\left\{\Gamma_{\chi \chi}^{H_{k}}\right\} \operatorname{Re}\left\{\Gamma_{q_{i} q_{i}}^{H_{k}}\right\}\left(\bar{u}_{3} u_{1}\right)\left(\bar{u}_{4} u_{2}\right)+\text { spin-dependent terms } .
\end{aligned}
$$

Evidently, the Wilson coefficient due to Higgs exchange is

$$
C_{q_{i}}^{h, H}=\frac{1}{2} \sum_{k=1}^{2} \frac{1}{m_{H_{k}}^{2}} \operatorname{Re}\left\{\Gamma_{\chi \chi}^{H_{k}}\right\} \operatorname{Re}\left\{\Gamma_{q_{i} q_{i}}^{H_{k}}\right\},
$$


and as in the case for squark exchange, we substitute the $H_{k}^{0} \chi \chi$ and $H_{k}^{0} q_{i} q_{i}$ couplings [103]

$$
\begin{aligned}
& \Gamma_{\chi \chi}^{H_{k}}=\frac{g_{2}}{c_{W}}\left(Z_{1 k}^{h} Z_{31}^{\chi *}-Z_{2 k}^{h} Z_{41}^{\chi *}\right)\left(Z_{11}^{\chi} s_{W}-Z_{21}^{\chi} c_{W}\right), \\
& \Gamma_{q_{i} q_{i}}^{H_{k}}=-\frac{Y_{q_{i}}}{\sqrt{2}} Z_{q k}^{h} \quad \text { where } Z_{q k}^{h}=\left\{\begin{array}{cc}
Z_{2 k}^{h} & \text { for } q=u \\
-Z_{1 k}^{h} & \text { for } q=d
\end{array},\right.
\end{aligned}
$$

to obtain the analytic expressions

$$
\begin{aligned}
\bar{m}_{u_{i}} C_{u_{i}}^{h, H}= & \frac{g_{2}}{2 \sqrt{2}} \operatorname{Re}\left\{Y_{u_{i}}\right\}\left[\operatorname{Re}\left\{Z_{41}^{\chi *}\left(Z_{11}^{\chi} \tan \theta_{W}-Z_{21}^{\chi}\right)\right\}\left(\frac{c_{\alpha}^{2}}{m_{h}^{2}}+\frac{s_{\alpha}^{2}}{m_{H}^{2}}\right)\right. \\
& \left.+\operatorname{Re}\left\{Z_{31}^{\chi *}\left(Z_{11}^{\chi} \tan \theta_{W}-Z_{21}^{\chi}\right)\right\} s_{\alpha} c_{\alpha}\left(\frac{1}{m_{h}^{2}}-\frac{1}{m_{H}^{2}}\right)\right] \\
= & \frac{g_{1}^{2}}{4} \frac{\bar{m}_{u_{i}}}{\left|M_{1}\right|^{2}-|\mu|^{2}}\left[\operatorname{Re}\left\{M_{1}^{*}+\mu \cot \beta\right\}\left(\frac{c_{\alpha}^{2}}{m_{h}^{2}}+\frac{s_{\alpha}^{2}}{m_{H}^{2}}\right)\right. \\
& \left.-\operatorname{Re}\left\{M_{1}^{*} \cot \beta+\mu\right\} s_{\alpha} c_{\alpha}\left(\frac{1}{m_{h}^{2}}-\frac{1}{m_{H}^{2}}\right)\right] \\
\bar{m}_{d_{i}} C_{d_{i}}^{h, H}= & \frac{g_{2}}{2 \sqrt{2}} \operatorname{Re}\left\{Y_{d_{i}}\right\}\left[\operatorname{Re}\left\{Z_{31}^{\chi *}\left(Z_{11}^{\chi} \tan \theta_{W}-Z_{21}^{\chi}\right)\right\}\left(\frac{s_{\alpha}^{2}}{m_{h}^{2}}+\frac{c_{\alpha}^{2}}{m_{H}^{2}}\right)\right. \\
& \left.+\operatorname{Re}\left\{Z_{41}^{\chi *}\left(Z_{11}^{\chi} \tan \theta_{W}-Z_{21}^{\chi}\right)\right\} s_{\alpha} c_{\alpha}\left(\frac{1}{m_{h}^{2}}-\frac{1}{m_{H}^{2}}\right)\right] \\
= & \frac{g_{1}^{2}}{4} \frac{\bar{m}_{d_{i}}}{\left|M_{1}\right|^{2}-|\mu|^{2}}\left[\operatorname{Re}\left\{M_{1}^{*}+\mu \tan \beta\right\}\left(\frac{s_{\alpha}^{2}}{m_{h}^{2}}+\frac{c_{\alpha}^{2}}{m_{H}^{2}}\right)\right. \\
& \left.-\operatorname{Re}\left\{M_{1}^{*} \tan \beta+\mu\right\} s_{\alpha} c_{\alpha}\left(\frac{1}{m_{h}^{2}}-\frac{1}{m_{H}^{2}}\right)\right] .
\end{aligned}
$$

Open Access. This article is distributed under the terms of the Creative Commons Attribution License (CC-BY 4.0), which permits any use, distribution and reproduction in any medium, provided the original author(s) and source are credited.

\section{References}

[1] A.H. Chamseddine, R.L. Arnowitt and P. Nath, Locally supersymmetric grand unification, Phys. Rev. Lett. 49 (1982) 970 [INSPIRE].

[2] P. Nath, R.L. Arnowitt and A.H. Chamseddine, Gravity induced symmetry breaking and ground state of local supersymmetric GUTs, Phys. Lett. B 121 (1983) 33 [inSPIRE].

[3] R. Barbieri, S. Ferrara and C.A. Savoy, Gauge models with spontaneously broken local supersymmetry, Phys. Lett. B 119 (1982) 343 [INSPIRE].

[4] L.J. Hall, J.D. Lykken and S. Weinberg, Supergravity as the messenger of supersymmetry breaking, Phys. Rev. D 27 (1983) 2359 [INSPIRE].

[5] O. Buchmueller et al., Higgs and supersymmetry, Eur. Phys. J. C 72 (2012) 2020 [arXiv:1112.3564] [INSPIRE].

[6] O. Buchmueller et al., The CMSSM and NUHM1 in light of $7 \mathrm{TeV} L H C, B_{s} \rightarrow \mu^{+} \mu^{-}$and XENON100 data, Eur. Phys. J. C 72 (2012) 2243 [arXiv:1207.7315] [INSPIRE]. 
[7] M.E. Cabrera, J.A. Casas and R.R. de Austri, The health of SUSY after the Higgs discovery and the XENON100 data, JHEP 07 (2013) 182 [arXiv:1212.4821] [INSPIRE].

[8] C. Strege et al., Global fits of the CMSSM and NUHM including the LHC Higgs discovery and new XENON100 constraints, JCAP 04 (2013) 013 [arXiv:1212.2636] [INSPIRE].

[9] A. Fowlie et al., The CMSSM favoring new territories: the impact of new LHC limits and a 125 GeV Higgs, Phys. Rev. D 86 (2012) 075010 [arXiv: 1206. 0264] [INSPIRE].

[10] O. Buchmueller et al., The CMSSM and NUHM1 after LHC run 1, Eur. Phys. J. C 74 (2014) 2922 [arXiv: 1312.5250] [INSPIRE].

[11] P. Bechtle et al., Constrained supersymmetry after two years of LHC data: a global view with Fittino, JHEP 06 (2012) 098 [arXiv:1204.4199] [INSPIRE].

[12] P. Bechtle et al., Constrained supersymmetry after the Higgs boson discovery: a global analysis with Fittino, PoS(EPS-HEP 2013) 313 [arXiv:1310.3045] [INSPIRE].

[13] MSSM Working Group collaboration, A. Djouadi et al., The Minimal Supersymmetric Standard Model: group summary report, hep-ph/9901246 [INSPIRE].

[14] C.F. Berger, J.S. Gainer, J.L. Hewett and T.G. Rizzo, Supersymmetry without prejudice, JHEP 02 (2009) 023 [arXiv:0812.0980] [InSPIRE].

[15] C. Boehm, P.S.B. Dev, A. Mazumdar and E. Pukartas, Naturalness of light neutralino dark matter in pMSSM after LHC, XENON100 and Planck data, JHEP 06 (2013) 113 [arXiv: 1303.5386] [INSPIRE].

[16] P. Grothaus, M. Lindner and Y. Takanishi, Naturalness of neutralino dark matter, JHEP 07 (2013) 094 [arXiv: 1207.4434] [INSPIRE].

[17] T. Han, Z. Liu and A. Natarajan, Dark matter and Higgs bosons in the MSSM, JHEP 11 (2013) 008 [arXiv: 1303.3040] [InSPIRE].

[18] C. Strege et al., Profile likelihood maps of a 15-dimensional MSSM, JHEP 09 (2014) 081 [arXiv: 1405.0622] [INSPIRE].

[19] SuperCDMS collaboration, R. Agnese et al., Search for low-mass weakly interacting massive particles with SuperCDMS, Phys. Rev. Lett. 112 (2014) 241302 [arXiv:1402.7137] [INSPIRE].

[20] XENON100 collaboration, E. Aprile et al., Dark matter results from 100 live days of XENON100 data, Phys. Rev. Lett. 107 (2011) 131302 [arXiv:1104.2549] [INSPIRE].

[21] LUX collaboration, D.S. Akerib et al., First results from the LUX dark matter experiment at the Sanford Underground Research Facility, Phys. Rev. Lett. 112 (2014) 091303 [arXiv: 1310.8214] [INSPIRE].

[22] XENON1T collaboration, E. Aprile, The XENON1T dark matter search experiment, Springer Proc. Phys. 148 (2013) 93 [arXiv:1206.6288] [InSPIRE].

[23] D.C. Malling et al., After LUX: the LZ program, arXiv:1110.0103 [INSPIRE].

[24] SuperCDMS collaboration, P.L. Brink, Conceptual design for SuperCDMS SNOLAB, J. Low. Temp. Phys. 167 (2012) 1093 [inSPIRE].

[25] J. Alwall, P. Schuster and N. Toro, Simplified models for a first characterization of new physics at the LHC, Phys. Rev. D 79 (2009) 075020 [arXiv: 0810.3921] [INSPIRE]. 
[26] LHC New Physics Working Group collaboration, D. Alves, Simplified models for LHC new physics searches, J. Phys. G 39 (2012) 105005 [arXiv: 1105. 2838] [INSPIRE].

[27] J. Barnard and B. Farmer, A simple technique for combining simplified models and its application to direct stop production, JHEP 06 (2014) 132 [arXiv:1402.3298] [INSPIRE].

[28] L. Edelhäuser, J. Heisig, M. Krämer, L. Oymanns and J. Sonneveld, Constraining supersymmetry at the LHC with simplified models for squark production, JHEP 12 (2014) 022 [arXiv: 1410.0965] [INSPIRE].

[29] L. Calibbi, J.M. Lindert, T. Ota and Y. Takanishi, LHC tests of light neutralino dark matter without light sfermions, JHEP 11 (2014) 106 [arXiv:1410.5730] [INSPIRE].

[30] C. Cheung, L.J. Hall, D. Pinner and J.T. Ruderman, Prospects and blind spots for neutralino dark matter, JHEP 05 (2013) 100 [arXiv:1211.4873] [INSPIRE].

[31] M.W. Goodman and E. Witten, Detectability of certain dark matter candidates, Phys. Rev. D 31 (1985) 3059 [INSPIRE].

[32] K. Griest, Cross-sections, relic abundance and detection rates for neutralino dark matter, Phys. Rev. D 38 (1988) 2357 [Erratum ibid. D 39 (1989) 3802] [INSPIRE].

[33] M. Srednicki and R. Watkins, Coherent couplings of neutralinos to nuclei from squark mixing, Phys. Lett. B 225 (1989) 140 [INSPIRE].

[34] G.F. Giudice and E. Roulet, Energetic neutrinos from supersymmetric dark matter, Nucl. Phys. B 316 (1989) 429 [INSPIRE].

[35] M.A. Shifman, A.I. Vainshtein and V.I. Zakharov, Remarks on Higgs boson interactions with nucleons, Phys. Lett. B 78 (1978) 443 [INSPIRE].

[36] M. Drees and M.M. Nojiri, New contributions to coherent neutralino-nucleus scattering, Phys. Rev. D 47 (1993) 4226 [hep-ph/9210272] [INSPIRE].

[37] J. Hisano, K. Ishiwata and N. Nagata, Direct search of dark matter in high-scale supersymmetry, Phys. Rev. D 87 (2013) 035020 [arXiv:1210.5985] [INSPIRE].

[38] P. Huang and C.E.M. Wagner, Blind spots for neutralino dark matter in the MSSM with an intermediate $m_{A}$, Phys. Rev. D 90 (2014) 015018 [arXiv:1404.0392] [InSPIRE].

[39] A. Anandakrishnan, B. Shakya and K. Sinha, Dark matter at the pseudoscalar Higgs resonance in the phenomenological MSSM and SUSY GUTs, Phys. Rev. D 91 (2015) 035029 [arXiv: 1410.0356] [INSPIRE].

[40] ATLAS collaboration, Search for squarks and gluinos with the ATLAS detector in final states with jets and missing transverse momentum using $\sqrt{s}=8 \mathrm{TeV}$ proton-proton collision data, JHEP 09 (2014) 176 [arXiv:1405.7875] [INSPIRE].

[41] CMS collaboration, Search for new physics in the multijet and missing transverse momentum final state in proton-proton collisions at $\sqrt{s}=8 \mathrm{TeV}$, JHEP 06 (2014) 055 [arXiv: 1402 .4770] [INSPIRE].

[42] Y. Nir and N. Seiberg, Should squarks be degenerate?, Phys. Lett. B 309 (1993) 337 [hep-ph/9304307] [INSPIRE].

[43] Y. Nir and G. Raz, Quark squark alignment revisited, Phys. Rev. D 66 (2002) 035007 [hep-ph/0206064] [INSPIRE]. 
[44] A. Crivellin and M. Davidkov, Do squarks have to be degenerate? Constraining the mass splitting with Kaon and D mixing, Phys. Rev. D 81 (2010) 095004 [arXiv:1002.2653] [INSPIRE].

[45] R. Mahbubani, M. Papucci, G. Perez, J.T. Ruderman and A. Weiler, Light nondegenerate squarks at the LHC, Phys. Rev. Lett. 110 (2013) 151804 [arXiv:1212.3328] [INSPIRE].

[46] S. Dimopoulos and G.F. Giudice, Naturalness constraints in supersymmetric theories with nonuniversal soft terms, Phys. Lett. B 357 (1995) 573 [hep-ph/9507282] [INSPIRE].

[47] G.F. Giudice and A. Romanino, Split supersymmetry, Nucl. Phys. B 699 (2004) 65 [Erratum ibid. B 706 (2005) 65] [hep-ph/0406088] [INSPIRE].

[48] R. Kitano and Y. Nomura, Supersymmetry, naturalness and signatures at the LHC, Phys. Rev. D 73 (2006) 095004 [hep-ph/0602096] [INSPIRE].

[49] M. Perelstein and C. Spethmann, A collider signature of the supersymmetric golden region, JHEP 04 (2007) 070 [hep-ph/0702038] [INSPIRE].

[50] C. Brust, A. Katz, S. Lawrence and R. Sundrum, SUSY, the third generation and the LHC, JHEP 03 (2012) 103 [arXiv:1110.6670] [InSPIRE].

[51] M. Papucci, J.T. Ruderman and A. Weiler, Natural SUSY endures, JHEP 09 (2012) 035 [arXiv:1110.6926] [INSPIRE].

[52] R. Barbieri and G.F. Giudice, Upper bounds on supersymmetric particle masses, Nucl. Phys. B 306 (1988) 63 [INSPIRE].

[53] A.G. Cohen, D.B. Kaplan and A.E. Nelson, The more minimal supersymmetric standard model, Phys. Lett. B 388 (1996) 588 [hep-ph/9607394] [INSPIRE].

[54] R. Barbieri and D. Pappadopulo, S-particles at their naturalness limits, JHEP 10 (2009) 061 [arXiv:0906.4546] [INSPIRE].

[55] J.R. Ellis, A. Ferstl and K.A. Olive, Reevaluation of the elastic scattering of supersymmetric dark matter, Phys. Lett. B 481 (2000) 304 [hep-ph/0001005] [INSPIRE].

[56] J.R. Ellis, A. Ferstl and K.A. Olive, Exploration of elastic scattering rates for supersymmetric dark matter, Phys. Rev. D 63 (2001) 065016 [hep-ph/0007113] [InSPIRE].

[57] H. Baer, A. Mustafayev, E.-K. Park and X. Tata, Target dark matter detection rates in models with a well-tempered neutralino, JCAP 01 (2007) 017 [hep-ph/0611387] [INSPIRE].

[58] V. Mandic, A. Pierce, P. Gondolo and H. Murayama, The lower bound on the neutralino nucleon cross-section, hep-ph/0008022 [INSPIRE].

[59] J.R. Ellis, K.A. Olive and C. Savage, Hadronic uncertainties in the elastic scattering of supersymmetric dark matter, Phys. Rev. D 77 (2008) 065026 [arXiv:0801.3656] [InSPIRE].

[60] A. Kurylov and M. Kamionkowski, Generalized analysis of weakly interacting massive particle searches, Phys. Rev. D 69 (2004) 063503 [hep-ph/0307185] [INSPIRE].

[61] F. Giuliani, Are direct search experiments sensitive to all spin-independent WIMP candidates?, Phys. Rev. Lett. 95 (2005) 101301 [hep-ph/0504157] [INSPIRE].

[62] S. Chang, J. Liu, A. Pierce, N. Weiner and I. Yavin, CoGeNT interpretations, JCAP 08 (2010) 018 [arXiv: 1004.0697] [InSPIRE].

[63] J.L. Feng, J. Kumar, D. Marfatia and D. Sanford, Isospin-violating dark matter, Phys. Lett. B 703 (2011) 124 [arXiv:1102.4331] [INSPIRE]. 
[64] V. Cirigliano, M.L. Graesser and G. Ovanesyan, WIMP-nucleus scattering in chiral effective theory, JHEP 10 (2012) 025 [arXiv:1205.2695] [INSPIRE].

[65] V. Cirigliano, M.L. Graesser, G. Ovanesyan and I.M. Shoemaker, Shining LUX on isospin-violating dark matter beyond leading order, Phys. Lett. B 739 (2014) 293 [arXiv:1311.5886] [INSPIRE].

[66] A. Crivellin, M. Hoferichter and M. Procura, Accurate evaluation of hadronic uncertainties in spin-independent WIMP-nucleon scattering: disentangling two- and three-flavor effects, Phys. Rev. D 89 (2014) 054021 [arXiv: 1312.4951] [INSPIRE].

[67] G. Bélanger, F. Boudjema, A. Pukhov and A. Semenov, MicrOMEGAs s: a program for calculating dark matter observables, Comput. Phys. Commun. 185 (2014) 960 [arXiv: 1305.0237] [INSPIRE].

[68] S.L. Adler, J.C. Collins and A. Duncan, Energy-momentum-tensor trace anomaly in spin 1/2 quantum electrodynamics, Phys. Rev. D 15 (1977) 1712 [INSPIRE].

[69] P. Minkowski, On the anomalous divergence of the dilatation current in gauge theories, PRINT-76-0813, University of Bern, Bern Switzerland (1976) [INSPIRE].

[70] N.K. Nielsen, The energy momentum tensor in a non-Abelian quark gluon theory, Nucl. Phys. B 120 (1977) 212 [INSPIRE].

[71] J.C. Collins, A. Duncan and S.D. Joglekar, Trace and dilatation anomalies in gauge theories, Phys. Rev. D 16 (1977) 438 [INSPIRE].

[72] M. Drees and M. Nojiri, Neutralino-nucleon scattering revisited, Phys. Rev. D 48 (1993) 3483 [hep-ph/9307208] [INSPIRE].

[73] A. Kryjevski, Heavy quark $\bar{q} q$ matrix elements in the nucleon from perturbative QCD, Phys. Rev. D 70 (2004) 094028 [hep-ph/0312196] [INSPIRE].

[74] L. Vecchi, WIMPs and un-naturalness, arXiv:1312.5695 [INSPIRE].

[75] R.J. Hill and M.P. Solon, Standard model anatomy of WIMP dark matter direct detection II: QCD analysis and hadronic matrix elements, Phys. Rev. D 91 (2015) 043505 [arXiv: 1409.8290] [INSPIRE].

[76] G. Prézeau, A. Kurylov, M. Kamionkowski and P. Vogel, New contribution to WIMP-nucleus scattering, Phys. Rev. Lett. 91 (2003) 231301 [astro-ph/0309115] [INSPIRE].

[77] M. Hoferichter, P. Klos and A. Schwenk, Chiral power counting of one- and two-body currents in direct detection of dark matter, Phys. Lett. B 746 (2015) 410 [arXiv: 1503.04811] [INSPIRE].

[78] A. Corsetti and P. Nath, Gaugino mass nonuniversality and dark matter in SUGRA, strings and D-brane models, Phys. Rev. D 64 (2001) 125010 [hep-ph/0003186] [INSPIRE].

[79] H.-Y. Cheng, Low-energy interactions of scalar and pseudoscalar Higgs bosons with baryons, Phys. Lett. B 219 (1989) 347 [INSPIRE].

[80] G. Colangelo et al., Review of lattice results concerning low energy particle physics, Eur. Phys. J. C 71 (2011) 1695 [arXiv:1011.4408] [INSPIRE].

[81] M. Frink and U.-G. Meißner, Chiral extrapolations of baryon masses for unquenched three flavor lattice simulations, JHEP 07 (2004) 028 [hep-lat/0404018] [INSPIRE]. 
[82] B.C. Tiburzi and A. Walker-Loud, Strong isospin breaking in the nucleon and delta masses, Nucl. Phys. A 764 (2006) 274 [hep-lat/0501018] [INSPIRE].

[83] R.D. Young, Strange quark content of the nucleon and dark matter searches, PoS (LATTICE 2012) 014 [arXiv: 1301.1765] [INSPIRE].

[84] A.S. Kronfeld, Twenty-first century lattice gauge theory: results from the QCD Lagrangian, Ann. Rev. Nucl. Part. Sci. 62 (2012) 265 [arXiv:1203.1204] [INSPIRE].

[85] P. Junnarkar and A. Walker-Loud, Scalar strange content of the nucleon from lattice QCD, Phys. Rev. D 87 (2013) 114510 [arXiv:1301.1114] [InSPIRE].

[86] T.P. Cheng and R.F. Dashen, Is $\mathrm{SU}(2) \times \mathrm{SU}(2)$ a better symmetry than $\mathrm{SU}(3)$ ?, Phys. Rev. Lett. 26 (1971) 594 [INSPIRE].

[87] M. Hoferichter, B. Kubis and U.-G. Meißner, Isospin breaking in the pion-nucleon scattering lengths, Phys. Lett. B 678 (2009) 65 [arXiv:0903.3890] [INSPIRE].

[88] M. Hoferichter, B. Kubis and U.G. Meißner, Isospin violation in low-energy pion-nucleon scattering revisited, Nucl. Phys. A 833 (2010) 18 [arXiv:0909.4390] [INSPIRE].

[89] D. Gotta et al., Pionic hydrogen, Lect. Notes Phys. 745 (2008) 165 [InSPIRE].

[90] T. Strauch et al., Pionic deuterium, Eur. Phys. J. A 47 (2011) 88 [arXiv:1011.2415] [INSPIRE].

[91] V. Baru, C. Hanhart, M. Hoferichter, B. Kubis, A. Nogga and D.R. Phillips, Precision calculation of the $\pi^{-}$deuteron scattering length and its impact on threshold $\pi-N$ scattering, Phys. Lett. B 694 (2011) 473 [arXiv:1003.4444] [INSPIRE].

[92] V. Baru, C. Hanhart, M. Hoferichter, B. Kubis, A. Nogga and D.R. Phillips, Precision calculation of threshold $\pi^{-} d$ scattering, $\pi N$ scattering lengths and the GMO sum rule, Nucl. Phys. A 872 (2011) 69 [arXiv:1107.5509] [INSPIRE].

[93] C. Ditsche, M. Hoferichter, B. Kubis and U.G. Meißner, Roy-Steiner equations for pion-nucleon scattering, JHEP 06 (2012) 043 [arXiv:1203.4758] [INSPIRE].

[94] M. Hoferichter, C. Ditsche, B. Kubis and U.G. Meißner, Dispersive analysis of the scalar form factor of the nucleon, JHEP 06 (2012) 063 [arXiv:1204.6251] [INSPIRE].

[95] M. Hoferichter, J.R. de Elvira, B. Kubis and U.-G. Meißner, Precision determination of the pion-nucleon $\sigma$-term from Roy-Steiner equations, arXiv:1506.04142 [INSPIRE].

[96] J. Gasser, H. Leutwyler and M.E. Sainio, Sigma term update, Phys. Lett. B 253 (1991) 252 [INSPIRE].

[97] M.M. Pavan, I.I. Strakovsky, R.L. Workman and R.A. Arndt, The pion nucleon $\Sigma$ term is definitely large: results from a G.W.U. analysis of $\pi$ nucleon scattering data, PiN Newslett. 16 (2002) 110 [hep-ph/0111066] [INSPIRE].

[98] J.M. Alarcón, J. Martin Camalich and J.A. Oller, The chiral representation of the $\pi N$ scattering amplitude and the pion-nucleon sigma term, Phys. Rev. D 85 (2012) 051503 [arXiv:1110.3797] [INSPIRE].

[99] J. Giedt, A.W. Thomas and R.D. Young, Dark matter, the CMSSM and lattice QCD, Phys. Rev. Lett. 103 (2009) 201802 [arXiv:0907.4177] [INSPIRE].

[100] B. Borasoy and U.-G. Meißner, Chiral expansion of baryon masses and $\sigma$-terms, Annals Phys. 254 (1997) 192 [hep-ph/9607432] [INSPIRE]. 
[101] P.E. Shanahan, A.W. Thomas and R.D. Young, Scale setting, sigma terms and the Feynman-Hellman theorem, PoS (LATTICE 2012) 165 [arXiv: 1301.3231] [INSPIRE].

[102] G. Bélanger, F. Boudjema, A. Pukhov and A. Semenov, Dark matter direct detection rate in a generic model with MicrOMEGAs 2.2, Comput. Phys. Commun. 180 (2009) 747 [arXiv: 0803.2360] [INSPIRE].

[103] J. Rosiek, Complete set of Feynman rules for the minimal supersymmetric extension of the standard model, Phys. Rev. D 41 (1990) 3464 [Erratum hep-ph/9511250] [INSPIRE].

[104] A. Crivellin and U. Nierste, Supersymmetric renormalisation of the CKM matrix and new constraints on the squark mass matrices, Phys. Rev. D 79 (2009) 035018 [arXiv:0810.1613] [INSPIRE].

[105] T. Falk, A. Ferstl and K.A. Olive, New contributions to neutralino elastic cross-sections from CP-violating phases in the MSSM, Phys. Rev. D 59 (1999) 055009 [Erratum ibid. D 60 (1999) 119904] [hep-ph/9806413] [INSPIRE].

[106] ATLAS collaboration, Observation of a new particle in the search for the standard model Higgs boson with the ATLAS detector at the LHC, Phys. Lett. B 716 (2012) 1 [arXiv:1207.7214] [INSPIRE].

[107] CMS collaboration, Observation of a new boson at a mass of $125 \mathrm{GeV}$ with the CMS experiment at the LHC, Phys. Lett. B 716 (2012) 30 [arXiv:1207.7235] [INSPIRE].

[108] R.L. Arnowitt and P. Nath, Predictions of neutralino dark matter event rates in minimal supergravity unification, Phys. Rev. D 54 (1996) 2374 [hep-ph/9509260] [INSPIRE].

[109] L. Hofer, U. Nierste and D. Scherer, Resummation of $\tan \beta$-enhanced supersymmetric loop corrections beyond the decoupling limit, JHEP 10 (2009) 081 [arXiv:0907.5408] [INSPIRE].

[110] A. Crivellin, L. Hofer and J. Rosiek, Complete resummation of chirally-enhanced loop-effects in the MSSM with non-minimal sources of flavor-violation, JHEP 07 (2011) 017 [arXiv: 1103.4272] [INSPIRE].

[111] M. Guchait, Exact solution of the neutralino mass matrix, Z. Phys. C 57 (1993) 157 [Erratum ibid. C 61 (1994) 178] [INSPIRE].

[112] M.M. El Kheishen, A.A. Aboshousha and A.A. Shafik, Analytic formulas for the neutralino masses and the neutralino mixing matrix, Phys. Rev. D 45 (1992) 4345 [INSPIRE].

[113] V.D. Barger, M.S. Berger and P. Ohmann, The supersymmetric particle spectrum, Phys. Rev. D 49 (1994) 4908 [hep-ph/9311269] [INSPIRE].

[114] L.J. Hall, R. Rattazzi and U. Sarid, The top quark mass in supersymmetric $\mathrm{SO}(10)$ unification, Phys. Rev. D 50 (1994) 7048 [hep-ph/9306309] [INSPIRE].

[115] M. Carena, M. Olechowski, S. Pokorski and C.E.M. Wagner, Electroweak symmetry breaking and bottom-top Yukawa unification, Nucl. Phys. B 426 (1994) 269 [hep-ph/9402253] [INSPIRE].

[116] M. Carena, D. Garcia, U. Nierste and C.E.M. Wagner, Effective Lagrangian for the $\bar{t} b H^{+}$ interaction in the MSSM and charged Higgs phenomenology, Nucl. Phys. B 577 (2000) 88 [hep-ph/9912516] [INSPIRE].

[117] T. Banks, Supersymmetry and the quark mass matrix, Nucl. Phys. B 303 (1988) 172 [INSPIRE]. 
[118] F. Borzumati, G.R. Farrar, N. Polonsky and S.D. Thomas, Soft Yukawa couplings in supersymmetric theories, Nucl. Phys. B 555 (1999) 53 [hep-ph/9902443] [INSPIRE].

[119] A. Crivellin and J. Girrbach, Constraining the MSSM sfermion mass matrices with light fermion masses, Phys. Rev. D 81 (2010) 076001 [arXiv: 1002.0227] [INSPIRE].

[120] A. Crivellin, Effective Higgs vertices in the generic MSSM, Phys. Rev. D 83 (2011) 056001 [arXiv: 1012.4840] [INSPIRE].

[121] A. Katz, M. Reece and A. Sajjad, Naturalness, $b \rightarrow s \gamma$ and SUSY heavy Higgses, JHEP 10 (2014) 102 [arXiv: 1406.1172] [INSPIRE].

[122] K. Griest and D. Seckel, Three exceptions in the calculation of relic abundances, Phys. Rev. D 43 (1991) 3191 [INSPIRE].

[123] J.R. Ellis, T. Falk and K.A. Olive, Neutralino-stau coannihilation and the cosmological upper limit on the mass of the lightest supersymmetric particle, Phys. Lett. B 444 (1998) 367 [hep-ph/9810360] [INSPIRE].

[124] J.R. Ellis, T. Falk, K.A. Olive and M. Srednicki, Calculations of neutralino-stau coannihilation channels and the cosmologically relevant region of MSSM parameter space, Astropart. Phys. 13 (2000) 181 [Erratum ibid. 15 (2001) 413] [hep-ph/9905481] [INSPIRE].

[125] Muon G-2 collaboration, G.W. Bennett et al., Final report of the muon E821 anomalous magnetic moment measurement at BNL, Phys. Rev. D 73 (2006) 072003 [hep-ex/0602035] [INSPIRE].

[126] F. Jegerlehner and A. Nyffeler, The muon g-2, Phys. Rept. 477 (2009) 1 [arXiv:0902.3360] [INSPIRE].

[127] J. Prades, E. de Rafael and A. Vainshtein, The hadronic light-by-light scattering contribution to the muon and electron anomalous magnetic moments, Adv. Ser. Direct. High Energy Phys. 20 (2009) 303 [arXiv:0901.0306] [INSPIRE].

[128] T. Aoyama, M. Hayakawa, T. Kinoshita and M. Nio, Complete tenth-order QED contribution to the muon g-2, Phys. Rev. Lett. 109 (2012) 111808 [arXiv:1205.5370] [INSPIRE].

[129] C. Gnendiger, D. Stöckinger and H. Stöckinger-Kim, The electroweak contributions to $(g-2)_{\mu}$ after the Higgs boson mass measurement, Phys. Rev. D 88 (2013) 053005 [arXiv: 1306.5546] [INSPIRE].

[130] A. Czarnecki, W.J. Marciano and A. Vainshtein, Refinements in electroweak contributions to the muon anomalous magnetic moment, Phys. Rev. D 67 (2003) 073006 [Erratum ibid. D 73 (2006) 119901] [hep-ph/0212229] [INSPIRE].

[131] A. Kurz, T. Liu, P. Marquard and M. Steinhauser, Hadronic contribution to the muon anomalous magnetic moment to next-to-next-to-leading order, Phys. Lett. B 734 (2014) 144 [arXiv: 1403.6400] [INSPIRE].

[132] G. Colangelo, M. Hoferichter, A. Nyffeler, M. Passera and P. Stoffer, Remarks on higher-order hadronic corrections to the muon g-2, Phys. Lett. B 735 (2014) 90 [arXiv: 1403.7512] [INSPIRE].

[133] M. Davier, A. Hoecker, B. Malaescu and Z. Zhang, Reevaluation of the hadronic contributions to the muon $g-2$ and to $\alpha\left(M_{Z}^{2}\right)$, Eur. Phys. J. C 71 (2011) 1515 [Erratum ibid. C 72 (2012) 1874] [arXiv: 1010.4180] [INSPIRE]. 
[134] K. Hagiwara, R. Liao, A.D. Martin, D. Nomura and T. Teubner, $(g-2)_{\mu}$ and $\alpha\left(M_{Z}^{2}\right)$ re-evaluated using new precise data, J. Phys. G 38 (2011) 085003 [arXiv:1105.3149] [INSPIRE].

[135] T. Blum et al., The muon $(g-2)$ theory value: present and future, arXiv:1311.2198 [INSPIRE].

[136] M. Benayoun et al., Hadronic contributions to the muon anomalous magnetic moment: strategies for improvements of the accuracy of the theoretical prediction, arXiv:1407.4021 [INSPIRE].

[137] G. Colangelo, M. Hoferichter, M. Procura and P. Stoffer, Dispersive approach to hadronic light-by-light scattering, JHEP 09 (2014) 091 [arXiv:1402.7081] [INSPIRE].

[138] G. Colangelo, M. Hoferichter, B. Kubis, M. Procura and P. Stoffer, Towards a data-driven analysis of hadronic light-by-light scattering, Phys. Lett. B 738 (2014) 6 [arXiv:1408.2517] [INSPIRE].

[139] V. Pauk and M. Vanderhaeghen, Anomalous magnetic moment of the muon in a dispersive approach, Phys. Rev. D 90 (2014) 113012 [arXiv:1409.0819] [INSPIRE].

[140] G. Colangelo, M. Hoferichter, M. Procura and P. Stoffer, Dispersion relation for hadronic light-by-light scattering: theoretical foundations, arXiv:1506.01386 [INSPIRE].

[141] T. Blum, S. Chowdhury, M. Hayakawa and T. Izubuchi, Hadronic light-by-light scattering contribution to the muon anomalous magnetic moment from lattice QCD, Phys. Rev. Lett. 114 (2015) 012001 [arXiv: 1407.2923] [INSPIRE].

[142] Muon G-2 collaboration, J. Grange et al., Muon $(g-2)$ technical design report, arXiv: 1501.06858 [INSPIRE].

[143] J-PARC G-2/EDM collaboration, N. Saito, A novel precision measurement of muon $g-2$ and EDM at J-PARC, AIP Conf. Proc. 1467 (2012) 45 [InSPIRE].

[144] D. Stöckinger, The muon magnetic moment and supersymmetry, J. Phys. G 34 (2007) R45 [hep-ph/0609168] [INSPIRE].

[145] G.-C. Cho, K. Hagiwara, Y. Matsumoto and D. Nomura, The MSSM confronts the precision electroweak data and the muon $g$ - 2, JHEP 11 (2011) 068 [arXiv:1104.1769] [INSPIRE].

[146] H.G. Fargnoli, C. Gnendiger, S. Paßehr, D. Stöckinger and H. Stöckinger-Kim, Non-decoupling two-loop corrections to $(g-2)_{\mu}$ from fermion/sfermion loops in the MSSM, Phys. Lett. B 726 (2013) 717 [arXiv:1309.0980] [INSPIRE].

[147] H. Fargnoli, C. Gnendiger, S. Paßehr, D. Stöckinger and H. Stöckinger-Kim, Two-loop corrections to the muon magnetic moment from fermion/sfermion loops in the MSSM: detailed results, JHEP 02 (2014) 070 [arXiv:1311.1775] [INSPIRE].

[148] A. Crivellin, J. Girrbach and U. Nierste, Yukawa coupling and anomalous magnetic moment of the muon: an update for the LHC era, Phys. Rev. D 83 (2011) 055009 [arXiv: 1010.4485] [INSPIRE].

[149] M. Endo, K. Hamaguchi, T. Kitahara and T. Yoshinaga, Probing bino contribution to muon $g-2$, JHEP 11 (2013) 013 [arXiv:1309.3065] [InSPIRE].

[150] CMS collaboration, Search for physics beyond the standard model in events with two opposite-sign same-flavor leptons, jets and missing transverse energy in pp collisions at $\sqrt{s}=8 \mathrm{TeV}$, CMS-PAS-SUS-12-019, CERN, Geneva Switzerland (2012). 
[151] A. Crivellin, M. Hoferichter and M. Procura, Improved predictions for $\mu \rightarrow e$ conversion in nuclei and Higgs-induced lepton flavor violation, Phys. Rev. D 89 (2014) 093024 [arXiv: 1404.7134] [INSPIRE].

[152] O. Buchmueller et al., The NUHM2 after LHC run 1, Eur. Phys. J. C 74 (2014) 3212 [arXiv:1408.4060] [INSPIRE].

[153] CMS collaboration, Search for MSSM neutral Higgs bosons decaying to tau pairs in pp collisions, CMS-PAS-HIG-13-021, CERN, Geneva Switzerland (2013).

[154] M. Misiak et al., Updated NNLO QCD predictions for the weak radiative B-meson decays, Phys. Rev. Lett. 114 (2015) 221801 [arXiv:1503.01789] [INSPIRE].

[155] P. Batra, A. Delgado, D.E. Kaplan and T.M.P. Tait, The Higgs mass bound in gauge extensions of the minimal supersymmetric standard model, JHEP 02 (2004) 043 [hep-ph/0309149] [INSPIRE].

[156] L.J. Hall, D. Pinner and J.T. Ruderman, A natural SUSY Higgs near 126 GeV, JHEP 04 (2012) 131 [arXiv: 1112.2703] [InSPIRE].

[157] ATLAS collaboration, Search for direct top squark pair production in final states with one isolated lepton, jets and missing transverse momentum in $\sqrt{s}=8 \mathrm{TeV}$ pp collisions using $21 \mathrm{fb}^{-1}$ of ATLAS data, ATLAS-CONF-2013-037, CERN, Geneva Switzerland (2013).

[158] J. Rosiek, P. Chankowski, A. Dedes, S. Jager and P. Tanedo, SUSY_FLAVOR: a computational tool for FCNC and CP-violating processes in the MSSM, Comput. Phys. Commun. 181 (2010) 2180 [arXiv:1003.4260] [INSPIRE].

[159] A. Crivellin, J. Rosiek, P.H. Chankowski, A. Dedes, S. Jaeger and P. Tanedo, SUSY_FLAVOR v2: a computational tool for FCNC and CP-violating processes in the MSSM, Comput. Phys. Commun. 184 (2013) 1004 [arXiv: 1203.5023] [INSPIRE].

[160] J. Rosiek, SUSY_FLAVOR v2.5: a computational tool for FCNC and CP-violating processes in the MSSM, Comput. Phys. Commun. 188 (2014) 208 [arXiv:1410.0606] [INSPIRE].

[161] Particle Data Group collaboration, J. Beringer et al., Review of particle physics (RPP), Phys. Rev. D 86 (2012) 010001 [inSPIRE].

[162] C. Bobeth, M. Gorbahn, T. Hermann, M. Misiak, E. Stamou and M. Steinhauser, $B_{s, d} \rightarrow \ell^{+} \ell^{-}$in the standard model with reduced theoretical uncertainty, Phys. Rev. Lett. 112 (2014) 101801 [arXiv:1311.0903] [INSPIRE].

[163] A. Drozd, B. Grzadkowski, J.F. Gunion and Y. Jiang, Extending two-Higgs-doublet models by a singlet scalar field - the case for dark matter, JHEP 11 (2014) 105 [arXiv: 1408.2106] [INSPIRE].

[164] M.W. Cahill-Rowley, J.L. Hewett, A. Ismail, M.E. Peskin and T.G. Rizzo, pMSSM benchmark models for Snowmass 2013, arXiv:1305.2419 [INSPIRE].

[165] A. Crivellin and C. Greub, Two-loop supersymmetric QCD corrections to Higgs-quark-quark couplings in the generic MSSM, Phys. Rev. D 87 (2013) 015013 [Erratum ibid. D 87 (2013) 079901] [arXiv: 1210.7453] [INSPIRE]. 